\title{
Synthesis of Aryl Ketoamides via Aryne Insertion Into Imides
}

\author{
Austin C. Wright, Christopher K. Haley, Guillaume LaPointe, and Brian M. Stoltz \\ The Warren and Katharine Schlinger Laboratory for Chemistry and Chemical Engineering, \\ Division of Chemistry and Chemical Engineering, California Institute of Technology, \\ Pasadena, California, 91125, United States
}

\section{Table of Contents}

Materials and Methods

Imide Synthesis and Characterization Data

Aryl Ketoamide Synthesis and Characterization Data

Quinolone Synthesis and Characterization Data

Preparatory HPLC Conditions

Notes \& References

NMR \& IR Spectra

\section{Materials and Methods}

Unless otherwise stated, reactions were performed in flame-dried glassware under nitrogen atmosphere using dry, deoxygenated solvents (distilled or passed over a column of activated alumina). Reaction temperatures were controlled by an IKAmag temperature modulator. Thin layer chromatography (TLC) was performed using E. Merck silica gel 60 F254 precoated plates $(0.25 \mathrm{~mm})$ and visualized by UV fluorescence quenching. SiliaFlash P60 Academic Silica gel (particle size $0.040-0.063 \mathrm{~mm}$ ) was used for flash chromatography. ${ }^{1} \mathrm{H}$ and ${ }^{13} \mathrm{C}$ NMR spectra were recorded either on a Bruker AV III HD spectrometer equipped with a Prodigy liquid nitrogen temperature cryoprobe $(400 \mathrm{MHz}$ and $101 \mathrm{MHz}$, respectively) or on a Varian Inova $500\left(500 \mathrm{MHz}\right.$ and $125 \mathrm{MHz}$, respectively) and are reported relative to $\mathrm{Me}_{4} \mathrm{Si}(\delta 0.0)$. IR spectra were recorded on a Perkin Elmer Paragon 1000 Spectrometer and are reported in frequency of absorption $\left(\mathrm{cm}^{-1}\right)$. Preparatory HPLC was performed using an Agilent 1100 Series HPLC utilizing a Zorbax XDB-C18 column purchased from Agilent Technologies. HRMS were acquired using an Agilent 6200 Series TOF with an Agilent G1978A Multimode source in electrospray ionization (ESI), atmospheric pressure chemical ionization (APCI) or mixed (MM) ionization mode or with a JEOL JMS-600H in fast atom bombardment (FAB+). 


\section{Imide Synthesis and Characterization Data}<smiles>CC(C)CCNC(=O)CC(C)C</smiles>

S1

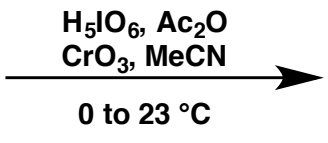

0 to $23^{\circ} \mathrm{C}$

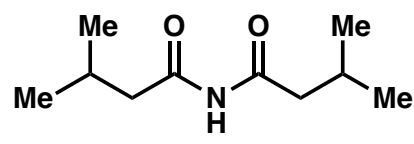

S2

3-methyl- $\boldsymbol{N}$-(3-methylbutanoyl)butanamide (S1): In air, $\mathrm{H}_{5} \mathrm{IO}_{6}(4.42 \mathrm{~g}, 19.4$ mmol, 6.0 equiv), $\mathrm{CrO}_{3}(16.2 \mathrm{mg}, 0.16 \mathrm{mmol}, 5.0 \mathrm{~mol} \%)$, and $\mathrm{MeCN}(46 \mathrm{~mL})$ were sequentially added to a round bottom flask equipped with a magnetic stir bar. The reaction mixture was stirred for 30 minutes at ambient temperature, at which point acetic anhydride $(1.8 \mathrm{~mL}, 19.4 \mathrm{mmol}, 6.0$ equiv) was added. The mixture was cooled to $0{ }^{\circ} \mathrm{C}$, and $\mathbf{S 1}(553 \mathrm{mg}, 3.23 \mathrm{mmol}, 1.0$ equiv) was added slowly. The resulting mixture was allowed to warm to ambient temperature over $12 \mathrm{~h}$. The reaction was quenched with ice water and extracted with EtOAc (4 x $40 \mathrm{~mL})$. The resulting organic layers were concentrated in vacuo and purified by column chromatography (20\% EtOAc in hexanes) to afford $\mathbf{S 2}\left(89 \mathrm{mg}, 15 \%\right.$ yield) as a white solid; $\mathrm{R}_{f}=0.15(10 \%$ EtOAc in hexanes); ${ }^{1} \mathrm{H} \mathrm{NMR}\left(400 \mathrm{MHz}, \mathrm{CDCl}_{3}\right) \delta 8.72$ (br s, 1H), 2.47 (d, $\left.J=9.4,4 \mathrm{H}\right), 2.14$ (heptet, $J=$ $9.0 \mathrm{~Hz}, 2 \mathrm{H}), 0.98(\mathrm{~s}, 12 \mathrm{H}) ;{ }^{13} \mathrm{C}\left(101 \mathrm{MHz} \mathrm{CDCl}_{3}\right) \delta$ 173.9, 46.3, 25.2, 22.4. IR (Neat Film, $\mathrm{NaCl}) 3272.4,3170.7,2957.7,2871.5,1728.6,1505.7,1466.8,1386.4,1367.0,1294.4,1246.8$, 1181.5, 1160.6, 1120.1, $1090.0 \mathrm{~cm}^{-1}$; HRMS (FAB+) $\mathrm{m} / z$ calc'd for $\mathrm{C}_{10} \mathrm{H}_{20} \mathrm{NO}_{2}[\mathrm{M}+\mathrm{H}]^{+}$: 186.1494 , found 186.1500 .

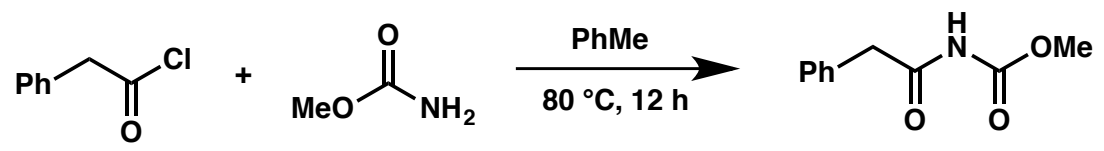

S3 S4

S5

Methyl (2-phenylacetyl)carbamate (S5): In air, 2-phenylacetyl chloride (S3, $309.2 \mathrm{mg}, 0.26$ $\mathrm{mL}, 2.00 \mathrm{mmol}, 1.0$ equiv), methyl carbamate (S4, $450.4 \mathrm{mg}, 6.00 \mathrm{mmol}, 3.0$ equiv), and PhMe $(10 \mathrm{~mL})$ were added. The reaction vessel was heated to $80^{\circ} \mathrm{C}$ for $12 \mathrm{~h}$. The reaction was cooled to $23{ }^{\circ} \mathrm{C}$, concentrated, and purified by column chromatography (10\% EtOAc in hexanes) to afford S5 (34.1 mg, 9\% yield) as white solid; $\mathrm{R}_{f}=0.40$ (50\% EtOAc in hexanes); ${ }^{1} \mathrm{H}$ NMR (500 $\left.\mathrm{MHz}, \mathrm{CDCl}_{3}\right) \delta 7.48$ (br s, 1H), 7.36-7.33 (m, 2H), 7.31-7.27 (m, 3H), 4.07 (s, 2H), 3.78 (s, $3 \mathrm{H}) ;{ }^{13} \mathrm{C}\left(126 \mathrm{MHz}, \mathrm{CDCl}_{3}\right) \delta 152.0,138.5,133.3,129.6,128.6,127.4,110.0,53.1$. IR (Neat Film, NaCl) 3246.1, 3172.3, 3014.0, 2259.7, 1788.1, 1757.5, 1686.2, 1520.2, 1455.7, 1257.2, 1216.1, 1192.1, 1144.1, 1049.9, 781.9, $705.3 \mathrm{~cm}^{-1}$; HRMS (ESI-APCI) $\mathrm{m} / z$ calc'd for $\mathrm{C}_{10} \mathrm{H}_{12} \mathrm{NO}_{3}[\mathrm{M}+\mathrm{H}]^{+}:$194.0817, found 194.0817. 


\section{Aryl Ketoamide Synthesis and Characterization Data}

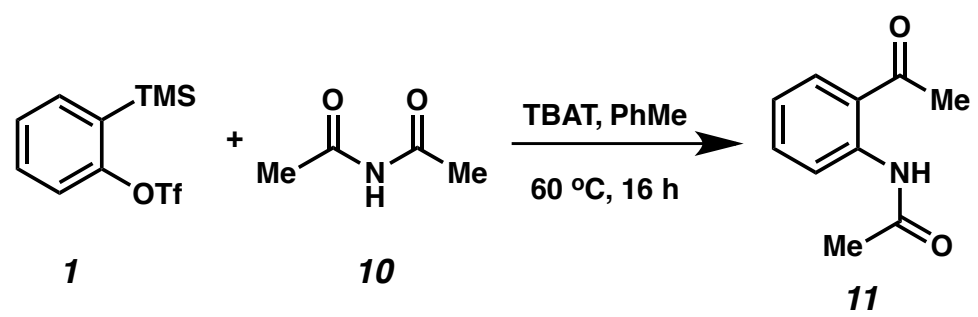

Representative Procedure for Acylamination

A 2-dram vial equipped with a magnetic stir bar was charged with TBAT (144.7 mg, 0.268 mmol, 2.0 equiv) and imide 10 (13.6 mg, $0.134 \mathrm{mmol}, 1.0$ equiv). The vial was purged with nitrogen, and PhMe (1.6 mL) was added via syringe followed by silyl triflate $1(60.0 \mathrm{mg}, 0.201$ mmol, 1.5 equiv). The vial was sealed and placed in an aluminum block preheated to $60{ }^{\circ} \mathrm{C}$. The reaction mixture was stirred at this temperature for $16 \mathrm{~h}$, then it was allowed to cool to $23{ }^{\circ} \mathrm{C}$. The mixture was concentrated in vacuo and purified by column chromatography (10\% EtOAc in hexanes) to afford 11 (23.6 mg, 89\% yield) as a white solid. Characterization data match those previously reported; ${ }^{1} \mathrm{H}$ NMR $\left(300 \mathrm{MHz}, \mathrm{CDCl}_{3}\right) \delta 11.70(\mathrm{~s}, 1 \mathrm{H}), 8.74(\mathrm{~d}, J=8.6 \mathrm{~Hz}$, $1 \mathrm{H}), 7.90(\mathrm{~d}, J=8.2 \mathrm{~Hz}, 1 \mathrm{H}), 7.56(\mathrm{dd}, J=7.3,8.6 \mathrm{~Hz}, 1 \mathrm{H}), 7.12(\mathrm{dd}, J=8.2,7.3 \mathrm{~Hz}, 1 \mathrm{H}), 2.67$ $(\mathrm{s}, 3 \mathrm{H}), 2.23(\mathrm{~s}, 3 \mathrm{H})$.

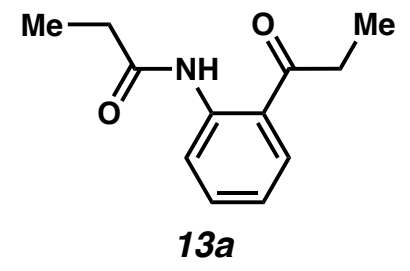

$N$-(2-propionylphenyl)propionamide (13a): Prepared according to the representative procedure using $N$-propionylpropionamide $(17.3 \mathrm{mg}, 0.134 \mathrm{mmol})$ and silyl triflate $1(60.0 \mathrm{mg}$, $0.201 \mathrm{mmol})$. The reaction was purified by column chromatography (10\% EtOAc in hexanes) to afford 13a (24.0 mg, 88\% yield) as a white solid. Characterization data match those previously reported. ${ }^{2}{ }^{1} \mathrm{H}$ NMR $\left(300 \mathrm{MHz}, \mathrm{CDCl}_{3}\right) \delta 11.78(\mathrm{~s}, 1 \mathrm{H}), 8.78(\mathrm{~d}, J=9.0 \mathrm{~Hz}, 1 \mathrm{H}), 7.93(\mathrm{~d}, J=9.0$ $\mathrm{Hz}, 1 \mathrm{H}), 7.57-7.51(\mathrm{~m}, 1 \mathrm{H}), 7.13-7.07(\mathrm{~m}, 1 \mathrm{H}), 3.06(\mathrm{q}, J=7.2 \mathrm{~Hz}, 2 \mathrm{H}), 2.50(\mathrm{q}, J=7.6,2 \mathrm{H})$, $1.31-1.20(\mathrm{~m}, 6 \mathrm{H})$.

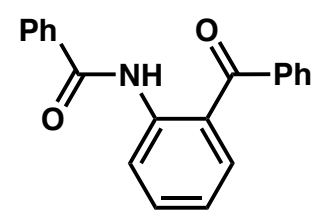

$13 b$

N-(2-benzoylphenyl)benzamide (13b): Prepared according to the representative procedure using $N$-benzoylbenzamide $(30.2 \mathrm{mg}, 0.134 \mathrm{mmol})$ and silyl triflate 1 (60.0 $\mathrm{mg}, 0.201 \mathrm{mmol})$. The reaction was purified by column chromatography (10\% EtOAc in hexanes) to afford 13b (27.4 mg, 68\% yield) as a white solid. Characterization data match those previously reported. ${ }^{3}$ 
${ }^{1} \mathrm{H}$ NMR $\left(400 \mathrm{MHz}, \mathrm{CDCl}_{3}\right) \delta 12.00(\mathrm{~s}, 1 \mathrm{H}), 8.92(\mathrm{~d}, J=8.2 \mathrm{~Hz}, 1 \mathrm{H}), 8.11-8.08(\mathrm{~m}, 2 \mathrm{H}), 7.74$ $(\mathrm{t}, J=4.3 \mathrm{~Hz}, 2 \mathrm{H}), 7.66-7.59(\mathrm{~m}, 3 \mathrm{H}), 7.56-7.49(\mathrm{~m}, 5 \mathrm{H}), 7.14(\mathrm{~s}, 1 \mathrm{H})$.

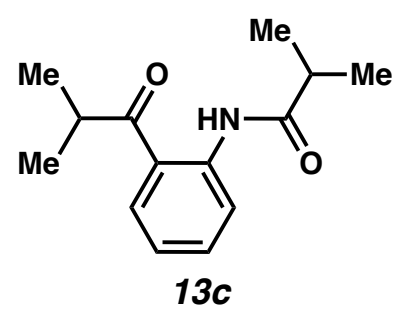

$N$-(2-isobutyrylphenyl)isobutyramide (13c): Prepared according to the representative procedure using $N$-isobutyrylisobutyramide $(23.6 \mathrm{mg}, 0.150 \mathrm{mmol})$ and silyl triflate $\mathbf{1}(67.1 \mathrm{mg}$, $0.225 \mathrm{mmol})$. Purification was achieved by prep HPLC to afford $13 \mathrm{c}(27.3 \mathrm{mg}, 78 \%$ yield) as a white solid; $\mathrm{R}_{f}=0.4\left(10 \%\right.$ EtOAc in hexanes); ${ }^{1} \mathrm{H}$ NMR $\left(400 \mathrm{MHz}, \mathrm{CDCl}_{3}\right) \delta 11.80(\mathrm{br} \mathrm{s}, 1 \mathrm{H})$, $8.79(\mathrm{~d}, J=6 \mathrm{~Hz}, 1 \mathrm{H}), 7.93(\mathrm{~d}, J=6 \mathrm{~Hz}, 1 \mathrm{H}), 7.53(\mathrm{t}, J=4.5 \mathrm{~Hz}, 1 \mathrm{H}), 7.10(\mathrm{t}, J=6 \mathrm{~Hz}, 1 \mathrm{H})$, 3.65 (sept, $J=6 \mathrm{~Hz}, 1 \mathrm{H}), 2.62$ (sept, $J=6 \mathrm{~Hz}, 1 \mathrm{H}), 1.28(\mathrm{~d}, J=6 \mathrm{~Hz}, 6 \mathrm{H}), 1.23(\mathrm{~d}, J=6.0 \mathrm{~Hz}$, $6 \mathrm{H}) ;{ }^{13} \mathrm{C}\left(101 \mathrm{MHz}, \mathrm{CDCl}_{3}\right) \delta 209.2,176.6,141.7,134.8,130.6,122.2,121.1,120.7,37.6,36.3$, 19.6 (2 unresolved signals); IR (Neat Film, NaCl) 3251.4, 2970.8, 2903.3, 2872.9, 1700.1, $1653.0,1604.8,1583.4,1521.7,1517.1,1467.7,1450.3,1383.3,1356.5,1350.9,1301.9$, 1239.0, 1211.6, 1157.4, 1099.6, 1083.7, 976.8, 755.1 cm $\mathrm{cm}^{-1}$; HRMS (MM: ESI-APCI) $\mathrm{m} / z$ calc'd for $\mathrm{C}_{14} \mathrm{H}_{20} \mathrm{NO}_{2}[\mathrm{M}+\mathrm{H}]^{+}:$234.1489, found: 234.1490.

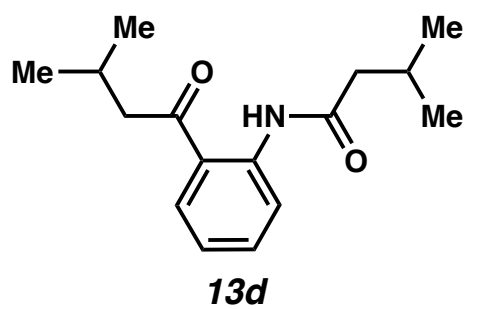

3-methyl- $N$-(2-(3-methylbutanoyl)phenyl)butanamide (13d): Prepared according to the representative procedure using imide $\mathbf{S 2}(37.1 \mathrm{mg}, 0.200 \mathrm{mmol})$ and silyl triflate $\mathbf{1}(89.5 \mathrm{mg}$, $0.300 \mathrm{mmol})$. Purification was achieved by prep HPLC to afford 13d $(41.3 \mathrm{mg}, 79 \%$ yield) as a white solid. $\mathrm{R}_{f}=0.30$ (10\% EtOAc in hexanes); ${ }^{1} \mathrm{H}$ NMR (400 MHz, $\left.\mathrm{CDCl}_{3}\right) \delta 11.73$ (br s, $\left.1 \mathrm{H}\right)$, $8.77(\mathrm{~d}, J=1.6 \mathrm{~Hz}, 1 \mathrm{H}), 7.90(\mathrm{~d}, J=10.8 \mathrm{~Hz}, 1 \mathrm{H}), 7.53(\mathrm{t}, J=9.8 \mathrm{~Hz}, 1 \mathrm{H}), 7.10(\mathrm{t}, J=9.8 \mathrm{~Hz}$, $1 \mathrm{H}), 2.88(\mathrm{~d}, J=9.2 \mathrm{~Hz}, 2 \mathrm{H}), 2.33-2.19(\mathrm{~m}, 4 \mathrm{H}), 1.03-0.99(\mathrm{~m}, 12 \mathrm{H}) ;{ }^{13} \mathrm{C}\left(126 \mathrm{MHz}, \mathrm{CDCl}_{3}\right)$ 205.0, 172.1, 141.0, 134.9, 130.4, 122.2, 121.92, 120.9, 49.0, 48.1, 26.3, 25.6, 22.7, 22.5. IR (Neat Film, NaCl) 3255.4, 2957.6, 2929.9, 2870.7, 1698.8, 1651.9, 1583.5, 1520.0, 1450.7, 1386.2, 1366.0, 1298.9, 1281.3, 1258.1, 1201.8, 1163.5, 1114.3, 1003.9, 947.5, 754.1 $\mathrm{cm}^{-1}$; HRMS (MM: ESI-APCI) $m / z$ calc'd for $\mathrm{C}_{16} \mathrm{H}_{24} \mathrm{NO}_{2}[\mathrm{M}+\mathrm{H}]^{+}: 262.1802$, found: 262.1804 . 
<smiles>COC(=O)c1ccccc1NC(=O)Cc1ccccc1</smiles>

$13 e$

Methyl 2-(2-phenylacetamido)benzoate (13e): Prepared according to the representative procedure using imide $\mathbf{S 5}(25.9 \mathrm{mg}, 0.134 \mathrm{mmol})$ and silyl triflate $\mathbf{1}(60.0 \mathrm{mg}, 0.201 \mathrm{mmol})$. The reaction was purified by column chromatography (10\% EtOAc in hexanes) to afford 13e (8.7 mg, 24\% yield) as a white solid. $\mathrm{R}_{f}=0.30\left(10 \%\right.$ EtOAc in hexanes); ${ }^{1} \mathrm{H}$ NMR (400 MHz, $\left.\mathrm{CDCl}_{3}\right) \delta 11.04(\mathrm{br} \mathrm{s}, 1 \mathrm{H}), 8.70(\mathrm{dd}, J=8.0 \mathrm{~Hz}, 1.2 \mathrm{~Hz}, 1 \mathrm{H}), 7.99(\mathrm{dd}, J=8.0,1.2 \mathrm{~Hz}, 1 \mathrm{H})$, 7.54-7.49 (m, 1H), 7.41-7.28 (m, 5H), 7.08-7.01 (m, 1H), 3.86 (s, 3H), 3.76 (s, 2H); ${ }^{13} \mathrm{C}(101$ $\left.\mathrm{MHz}, \mathrm{CDCl}_{3}\right)$ 170.0, 168.5, 141.4, 134.6, 134.4, 130.8, 129.5, 129.3, 128.9, 127.3, 122.6, 120.4, 52.3, 45.9. IR (Neat Film, NaCl) 2917.9, 1687.6, 1588.4, 1523.0, 1448.6, 1309.3, 1263.3, 1193.6, 1088.9, $756.5 \mathrm{~cm}^{-1}$; HRMS (MM: ESI-APCI) $\mathrm{m} / z$ calc'd for $\mathrm{C}_{16} \mathrm{H}_{15} \mathrm{NO}_{3}[\mathrm{M}+\mathrm{H}]^{+}$: 270.1125, found: 270.1129 .<smiles>CC(=O)Oc1ccccc1C(C)=O</smiles>

$13 g$

2-Acetylphenyl acetate (13g): Prepared according to the representative procedure using acetic anhydride $(13.7 \mathrm{mg}, 0.134 \mathrm{mmol})$ and silyl triflate $\mathbf{1}(60.0 \mathrm{mg}, 0.201 \mathrm{mmol})$. The reaction was purified by column chromatography (10\% EtOAc in hexanes) to afford $\mathbf{1 3 g}(12.8 \mathrm{mg}, 54 \%$ yield) as a white solid. Characterization data match those previously reported. ${ }^{4}{ }^{1} \mathrm{H}$ NMR (300 $\left.\mathrm{MHz}, \mathrm{CDCl}_{3}\right) \delta 7.78(\mathrm{~d}, J=7.8 \mathrm{~Hz}, 1 \mathrm{H}), 7.50(\mathrm{t}, J=7.7 \mathrm{~Hz}, 1 \mathrm{H}), 7.28(\mathrm{t}, J=7.6 \mathrm{~Hz}, 1 \mathrm{H}), 7.10$ $(\mathrm{d}, J=8.0 \mathrm{~Hz}, 1 \mathrm{H}), 2.52(\mathrm{~s}, 3 \mathrm{H}), 2.32(\mathrm{~s}, 3 \mathrm{H})$.<smiles>CC(=O)Nc1cc(C)c(C)cc1C(C)=O</smiles>

$15 a$

$N$-(2-acetyl-4,5-dimethylphenyl)acetamide (15a): Prepared according to the representative procedure using imide $10(8.4 \mathrm{mg}, 0.083 \mathrm{mmol})$ and silyl triflate $14 \mathbf{a}(40.5 \mathrm{mg}, 0.124 \mathrm{mmol})$. Purified by column chromatography (10\% EtOAc in hexanes) to afford $\mathbf{1 5 a}(6.5 \mathrm{mg}, 31 \%$ yield) as a white solid. $\mathrm{R}_{f}=0.15$ (10\% EtOAc in hexanes); $\left.{ }^{1} \mathrm{H} \mathrm{NMR} \mathrm{(400} \mathrm{MHz,} \mathrm{CDCl}_{3}\right) \delta 11.63$ (br s, $1 \mathrm{H}), 8.53(\mathrm{~s}, 1 \mathrm{H}), 7.61(\mathrm{~s}, 1 \mathrm{H}), 2.63(\mathrm{~s}, 3 \mathrm{H}), 2.31(\mathrm{~s}, 3 \mathrm{H}), 2.26(\mathrm{~s}, 3 \mathrm{H}), 2.21(\mathrm{~s}, 3 \mathrm{H}) ;{ }^{13} \mathrm{C}(101$ $\left.\mathrm{MHz}, \mathrm{CDCl}_{3}\right) \delta 202.4,169.4,145.3,139.1,132.4,130.6,121.5,119.8,28.6,25.6,20.6,19.4$. IR (Neat Film, NaCl) 3238.4, 2917.3, 1692.5, 1643.4, 1579.0, 1514.1, 1450.2, 1397.3, 1353.6, 
1286.8, 1270.0, 1235.1, 1018.7, 876.4, 758.9, $659.3 \mathrm{~cm}^{-1}$; HRMS (MM: ESI-APCI) $\mathrm{m} / z$ calc'd for $\mathrm{C}_{12} \mathrm{H}_{16} \mathrm{NO}_{2}[\mathrm{M}+\mathrm{H}]^{+}:$206.1176, found: 206.1171 .<smiles>CC(=O)Nc1cc2ccccc2cc1C(C)=O</smiles>

$15 b$

$N$-(3-acetylnaphthalen-2-yl)acetamide (15b): Prepared according to the representative procedure using imide $\mathbf{1 0}(29.0 \mathrm{mg}, 0.287 \mathrm{mmol})$ and silyl triflate $\mathbf{1 4 b}(150.0 \mathrm{mg}, 0.431 \mathrm{mmol})$. Purification was achieved by prep HPLC to afford $\mathbf{1 5 b}\left(28.7 \mathrm{mg}, 44 \%\right.$ yield) as a white solid; $\mathbf{R}_{f}$ $=0.35\left(30 \%\right.$ EtOAc in hexanes); ${ }^{1} \mathrm{H}$ NMR $\left(400 \mathrm{MHz}, \mathrm{CDCl}_{3}\right) \delta 11.50(\mathrm{br} \mathrm{s}, 1 \mathrm{H}), 9.12,(\mathrm{~s}, 1 \mathrm{H})$, $8.44(\mathrm{~s}, 1 \mathrm{H}), 7.83(\mathrm{~d}, J=9.0 \mathrm{~Hz}, 2 \mathrm{H}), 7.57(\mathrm{t}, J=7.5 \mathrm{~Hz}, 1 \mathrm{H}), 7.43(\mathrm{t}, J=7.5 \mathrm{~Hz}, 1 \mathrm{H}), 2.80(\mathrm{~s}$, $3 \mathrm{H}), 2.27(\mathrm{~s}, 3 \mathrm{H}) ;{ }^{13} \mathrm{C}\left(101 \mathrm{MHz}, \mathrm{CDCl}_{3}\right)$ 203.0, 169.3, 136.7, 136.1, 134.3, 129.7, 128.9, 128.2, 127.7, 125.6, 122.7, 117.7, 28.7, 25.6; IR (Neat Film, NaCl) 3217.8, 1682.2, 1654.4, 1577.0, 1546.1, 1480.7, 1437.2, 1352.5, 1386.4, 1286.3, 1277.9, 1203.7, 1148.0, 1020.5, 953.6, 885.1, 742.1, $656.2 \mathrm{~cm}^{-1}$; HRMS (MM: ESI-APCI) $\mathrm{m} / z$ calc'd for $\mathrm{C}_{14} \mathrm{H}_{14} \mathrm{NO}_{2}[\mathrm{M}+\mathrm{H}]^{+}: 228.1019$, found: 228.1022 .

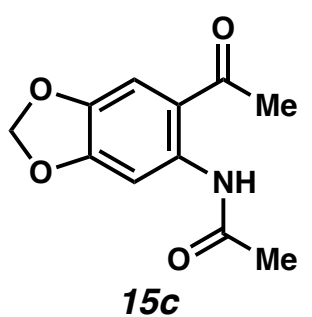

$N$-(6-acetylbenzo $[\boldsymbol{d}][\mathbf{1 , 3}]$ dioxol-5-yl)acetamide $(\mathbf{1 5 c})$ : Prepared according to the the representative procedure using imide $\mathbf{1 0}(20.4 \mathrm{mg}, 0.201 \mathrm{mmol})$ and silyl triflate $\mathbf{1 4 c}(103.4 \mathrm{mg}$, $0.302 \mathrm{mmol})$. Purification was achieved by prep HPLC to afford $\mathbf{1 5 c}(28.9 \mathrm{mg}, 65 \%$ yield $)$ as a white solid; $\mathrm{R}_{f}=0.55\left(50 \%\right.$ EtOAc in hexanes); ${ }^{1} \mathrm{H}$ NMR $\left(400 \mathrm{MHz}, \mathrm{CDCl}_{3}\right) \delta 12.08$ (br s, $\left.1 \mathrm{H}\right)$, $8.37(\mathrm{~s}, 1 \mathrm{H}), 7.25(\mathrm{~s}, 1 \mathrm{H}), 6.02(\mathrm{~s}, 2 \mathrm{H}), 2.57(\mathrm{~s}, 3 \mathrm{H}), 2.20(\mathrm{~s}, 3 \mathrm{H}),{ }^{13} \mathrm{C} \mathrm{NMR}(75 \mathrm{MHz}, \mathrm{CDCl} 3) \delta$ 200.6, 169.5, 152.9, 142.4, 139.3, 115.1, 109.6, 102.1, 101.5, 28.8, 25.6. IR (Neat Film, NaCl) 2916.7, 1692.6, 1611.8, 1502.7, 1483.5, 1433.8, 1370.2, 1342.5, 1243.2, 1178.9, 1118.8, 1044.8, 927.0 $\mathrm{cm}^{-1}$; HRMS (MM: ESI-APCI) $\mathrm{m} / z$ calc'd for $\mathrm{C}_{11} \mathrm{H}_{12} \mathrm{NO}_{4}[\mathrm{M}+\mathrm{H}]^{+}: 222.0761$, found: 222.0766 . 
<smiles>COc1cccc(NC(C)=O)c1C(C)=O</smiles>

$15 d$

$N$-(2-acetyl-3-methoxyphenol)acetamide (15d): Prepared according to the representative procedure using imide $10(13.6 \mathrm{mg}, 0.134 \mathrm{mmol})$ and silyl triflate $\mathbf{1 4 d}(66.0 \mathrm{mg}, 0.201 \mathrm{mmol})$. The reaction was purified by column chromatography (10\% EtOAc in hexanes) to afford 15d (13.0 mg, 47\% yield) as a white solid; $\mathrm{R}_{f}=0.20$ (20\% EtOAc in hexanes); ${ }^{1} \mathrm{H}$ NMR (500 MHz, $\left.\mathrm{CDCl}_{3}\right) \delta 10.49(\mathrm{~s}, 1 \mathrm{H}), 8.07(\mathrm{~d}, J=8.3 \mathrm{~Hz}, 1 \mathrm{H}), 7.40(\mathrm{td}, J=8.4,0.5 \mathrm{~Hz}, 1 \mathrm{H}), 6.69(\mathrm{dd}, J=$ 8.4, $0.9 \mathrm{~Hz}, 1 \mathrm{H}), 2.57$ (s, 3H), $3.90(\mathrm{~s}, 3 \mathrm{H}), 2.61(\mathrm{~s}, 3 \mathrm{H}) ;{ }^{13} \mathrm{C} \mathrm{NMR}\left(126 \mathrm{MHz}, \mathrm{CDCl}_{3}\right) \delta 204.6$, 169.2, 160.0, 139.2, 133.7, 116.5, 114.0, 106.2, 55.8, 33.7, 25.5; IR (Neat Film, NaCl) 3086.8, 2947.7, 1698.6, 1639.6, 1634.0, 1528.8, 1470.6, 1403.5, 1273.1, 1243.4, 1195.9, 1093.2, 1017.3, 967.4, 802.3, 735.2, $610.8 \mathrm{~cm}^{-1}$; HRMS (MM: ESI-APCI) $\mathrm{m} / z$ calc'd for $\mathrm{C}_{11} \mathrm{H}_{14} \mathrm{NO}_{3}$ $[\mathrm{M}+\mathrm{H}]^{+}: 262.1802$, found: 262.1804 .

\section{Quinolone Synthesis and Characterization Data}

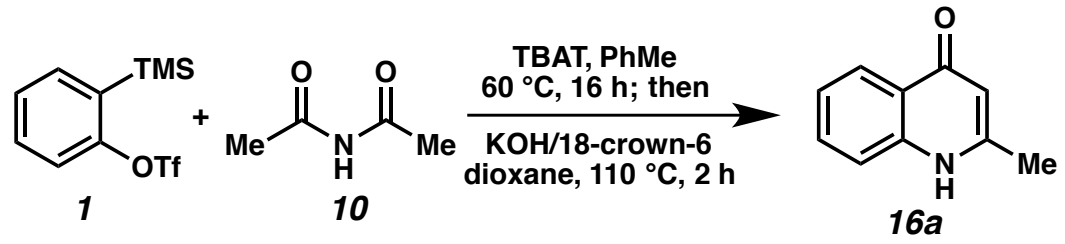

Representative Procedure for Quinolone Synthesis

A 2-dram vial equipped with a magnetic stir bar was charged with TBAT (144.7 mg, 0.268 mmol, 2.0 equiv) and imide 10 (13.6 mg, $0.134 \mathrm{mmol}, 1.0$ equiv). The vial was purged with nitrogen, and PhMe (1.6 mL) was added via syringe followed by silyl triflate $1(60.0 \mathrm{mg}, 0.201$ mmol, 1.5 equiv). The vial was sealed and placed in an aluminum block preheated to $60{ }^{\circ} \mathrm{C}$. The reaction mixture was stirred at this temperature for $16 \mathrm{~h}$, then it was allowed to cool to $23^{\circ} \mathrm{C}$. The mixture was concentrated in vacuo and then charged with dioxane $(1.6 \mathrm{~mL}), \mathrm{KOH}(22.6$ $\mathrm{mg}, 0.402 \mathrm{mmol}, 3.0$ equiv), and 18 -crown-6 (106.3 mg, $0.402 \mathrm{mmol}, 3.0$ equiv). The reaction vial was sealed and heated to $110{ }^{\circ} \mathrm{C}$ and stirred for $2 \mathrm{~h}$. The reaction was allowed to cool, diluted with $\mathrm{CH}_{2} \mathrm{Cl}_{2}(10 \mathrm{~mL})$, neutralized to $\mathrm{pH} \sim 7$, and washed with brine $(10 \mathrm{~mL})$. The layers were separated, and the aqueous layer was back extracted with $\mathrm{CH}_{2} \mathrm{Cl}_{2}(2 \times 10 \mathrm{~mL})$. The combined organic layers were dried $\left(\mathrm{Na}_{2} \mathrm{SO}_{4}\right)$, concentrated, and purified by column chromatography $\left(\mathrm{CH}_{2} \mathrm{Cl}_{2}\right.$ to $5 \% \mathrm{MeOH}$ in $\left.\mathrm{CH}_{2} \mathrm{Cl}_{2}\right)$ to provide $\mathbf{1 6 a}(15.1 \mathrm{mg}, 71 \%$ yield) as a yellow solid. Characterization data match those previously reported. ${ }^{5}{ }^{1} \mathrm{H}$ NMR $(400 \mathrm{MHz}$, $\left.\mathrm{CDCl}_{3}\right) \delta 12.04(\mathrm{~s}, 1 \mathrm{H}), 7.79(\mathrm{~d}, J=10.8 \mathrm{~Hz}, 1 \mathrm{H}), 7.65-7.62(\mathrm{~m}, 2 \mathrm{H}), 7.40-7.36(\mathrm{~m}, 1 \mathrm{H}), 6.74$ $(\mathrm{s}, 1 \mathrm{H}), 2.61(\mathrm{~d}, J=1.4 \mathrm{~Hz}, 3 \mathrm{H})$. 


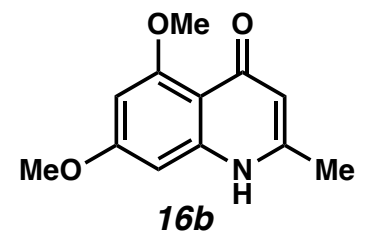

6,8-Dimethoxy-3-methylnaphthalen-1-(4H)-one (16b): Prepared according to the representative procedure using imide $10(13.6 \mathrm{mg}, 0.134 \mathrm{mmol})$ and 3,5-dimethoxy-2(trimethylsilyl)phenyl trifluoromethanesulfonate $(\mathbf{S 2}, 66.0 \mathrm{mg}, 0.201 \mathrm{mmol})$ to provide quinolone 16b (11.8 $\mathrm{mg}, 40 \%$ yield) as a brown solid. Characterization data match those previously reported. ${ }^{6}{ }^{1} \mathrm{H}$ NMR $\left(400 \mathrm{MHz}, \mathrm{CDCl}_{3}\right) \delta 7.08(\mathrm{~s}, 1 \mathrm{H}), 6.75(\mathrm{~s}, 1 \mathrm{H}), 6.74(\mathrm{~s}, 1 \mathrm{H})$, 6.24-6.23 (m, 1H), $3.78(\mathrm{~s}, 6 \mathrm{H}), 2.17(\mathrm{~s}, 3 \mathrm{H})$.

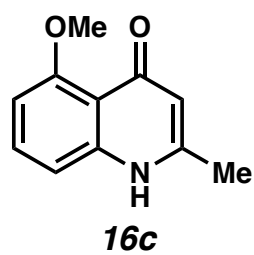

8-Methoxy-3-methylnaphthalen-1-(4H)-one (16c): Prepared according to the representative procedure using imide $\mathbf{1 0}(13.6 \mathrm{mg}, 0.134 \mathrm{mmol})$ and silyl triflate $\mathbf{1 3 d}(66.0 \mathrm{mg}, 0.201 \mathrm{mmol})$ to provide quinolone 16c $\left(10.6 \mathrm{mg}, 42 \%\right.$ yield) as a beige solid. $\mathrm{R}_{f}=0.35(10 \% \mathrm{MeOH}$ in $\left.\mathrm{CH}_{2} \mathrm{Cl}_{2}\right) ;{ }^{1} \mathrm{H}$ NMR $\left(400 \mathrm{MHz}, \mathrm{CDCl}_{3}\right) \delta 7.29-7.27(\mathrm{~m}, 1 \mathrm{H}), 7.21(\mathrm{t}, J=8.2,1 \mathrm{H}), 7.11$ (br s, $1 \mathrm{H}), 6.95(\mathrm{~d}, J=8.0 \mathrm{~Hz}, 1 \mathrm{H}), 6.67(\mathrm{dd}, J=12.0,11.2 \mathrm{~Hz}, 1 \mathrm{H}), 3.81(\mathrm{~s}, 3 \mathrm{H}), 2.18(\mathrm{~s}, 3 \mathrm{H}) ;{ }^{13} \mathrm{C}$ $\left(126 \mathrm{MHz}, \mathrm{CDCl}_{3}\right)$ 168.2, 160.2, 139.1, 134.8, 129.7, 111.8, 110.1, 105.6, 100.0 55.3, 24.8. IR (Neat Film, NaCl) 2920.7, 1664.8, 1598.3, 1548.7, 1492.8, 1425.9, 1369.9, 1252.6, 1156.1, 1044.1, $775.1 \mathrm{~cm}^{-1}$; HRMS (MM: ESI-APCI) $\mathrm{m} / z$ calc'd for $\mathrm{C}_{11} \mathrm{H}_{12} \mathrm{NO}_{2}[\mathrm{M}+\mathrm{H}]^{+}: 190.0863$, found: 190.0866 . 
Preparatory HPLC Conditions

Entry

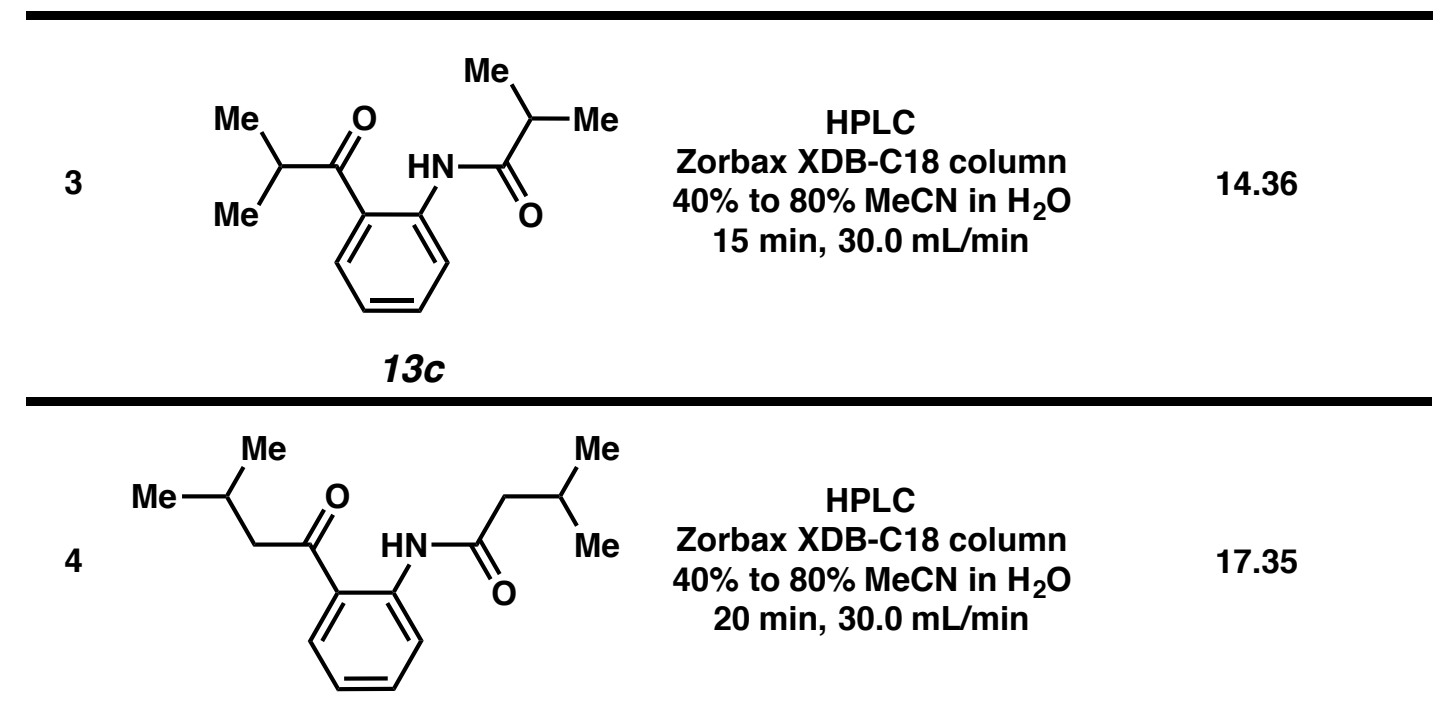

$13 d$<smiles>COC(=O)c1ccccc1NC(=O)Cc1ccccc1</smiles>

HPLC

Zorbax XDB-C18 column $0 \%$ to $80 \% \mathrm{MeCN}$ in $\mathrm{H}_{2} \mathrm{O}$ $10 \mathrm{~min}, 15 \mathrm{~mL} / \mathrm{min}$

$13 e$ 


\section{Notes \& References}

(1) Tobisu, M.; Ano, Y.; Chatani, N. Org. Lett. 2009, 11, 3250-3252.

(2) Canonne, P.; Boulanger, R.; Chantegrel, B. Tetrahedron 1987, 43, 663-668.

(3) Yin, Z.; Sun, P. Org. Lett. 2012, 77, 11339-11344.

(4) Rodriguez-Ramos, F.; Navarette, A.; Gonzalez-Andrade, M.; Alarcon, C.; Aguilera-Cruz, A.; Reyes-Ramirez, Adelfo. Bioorg. Chem. 2013, 50, 17-25.

(5) Cross, R. M.; Manetsch, R. J. Org. Chem. 2010, 75, 8654-8657.

(6) Liu, G.-B.; Xu, J.-L.; He, C.-C.; Chen, G.; Xu, Q.; Xu, H.-X.; Li, J.-X. Bioorg. Med. Chem. 2009, 17, 5433-5441. 


\section{NMR and IR Spectra}

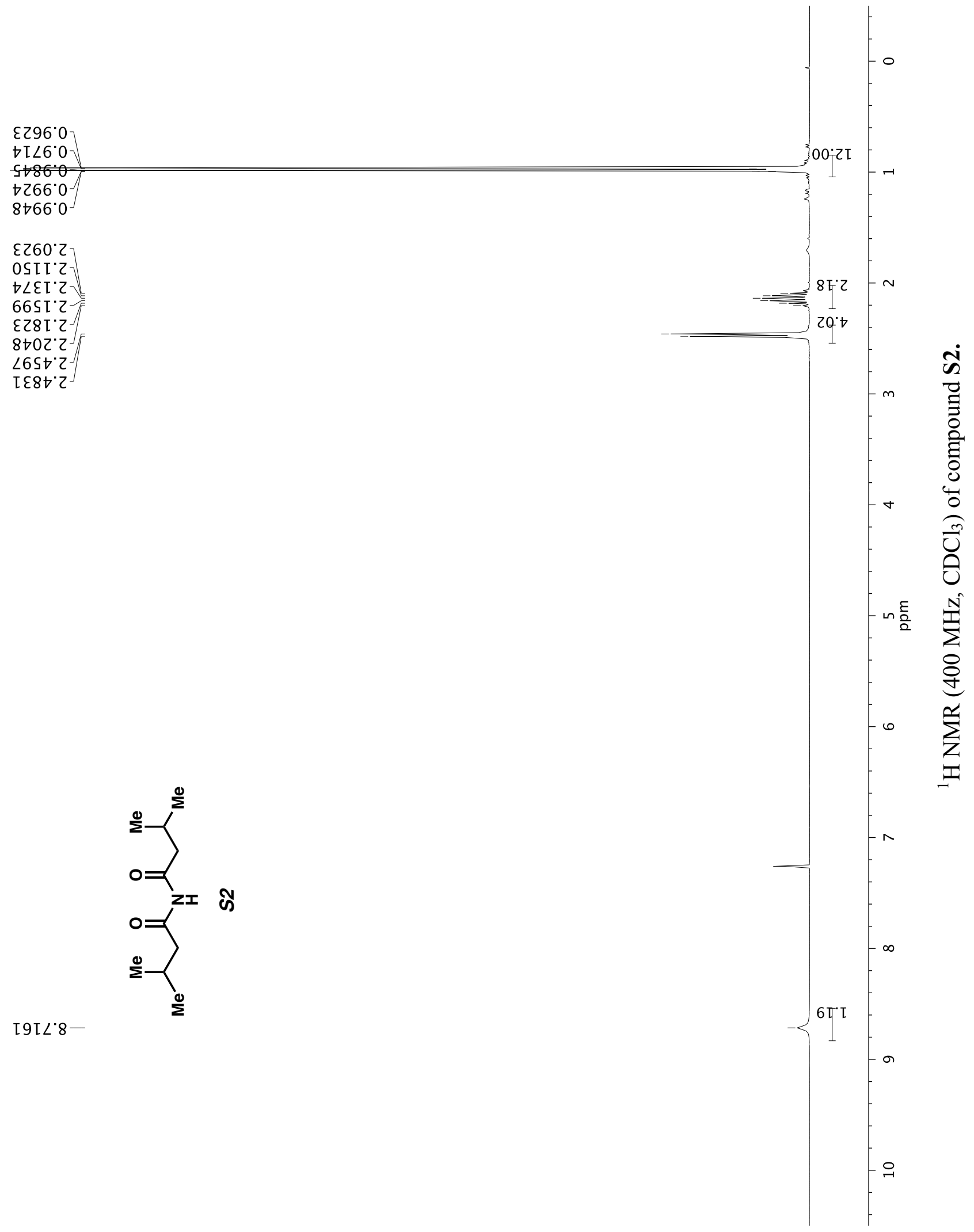



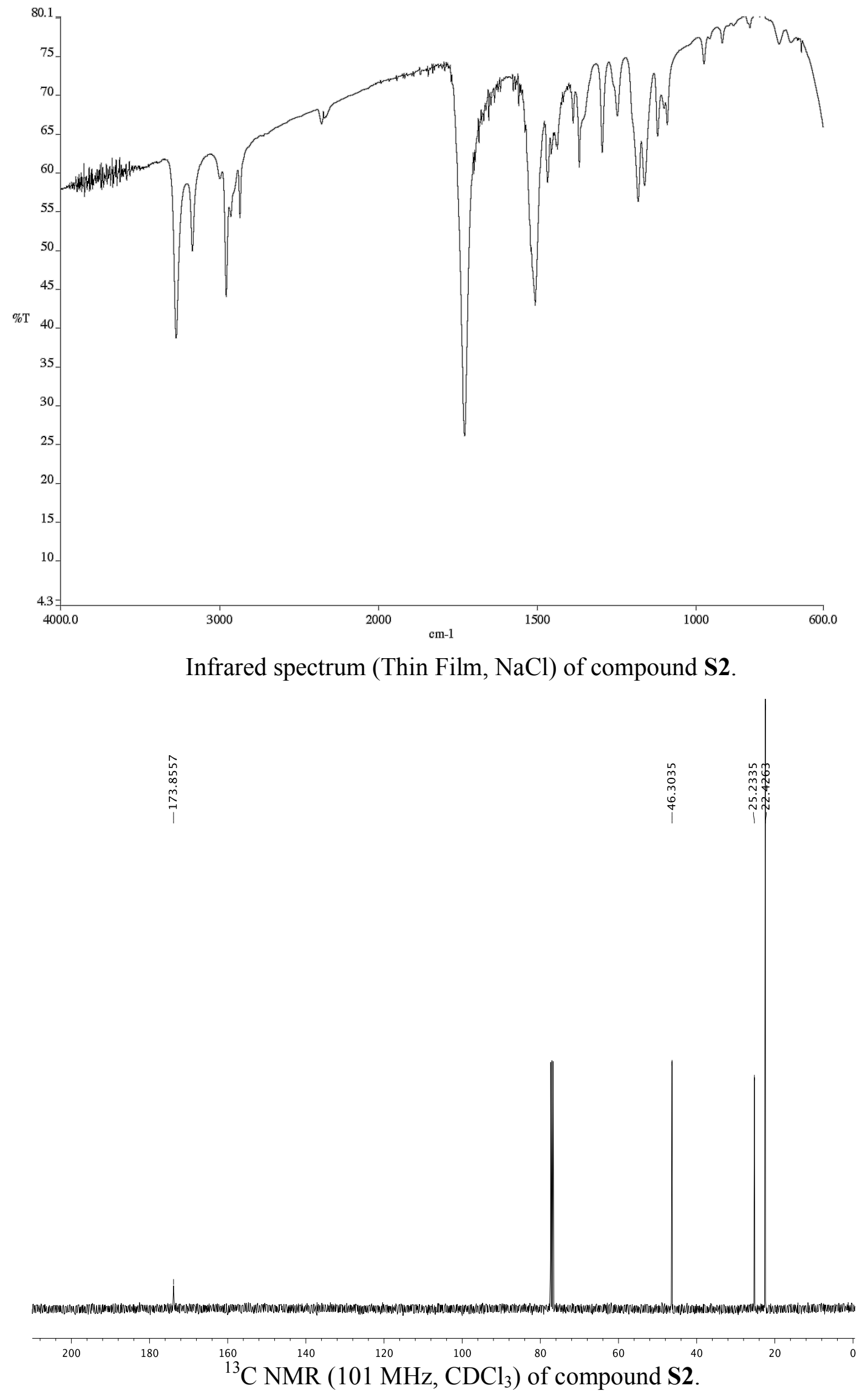


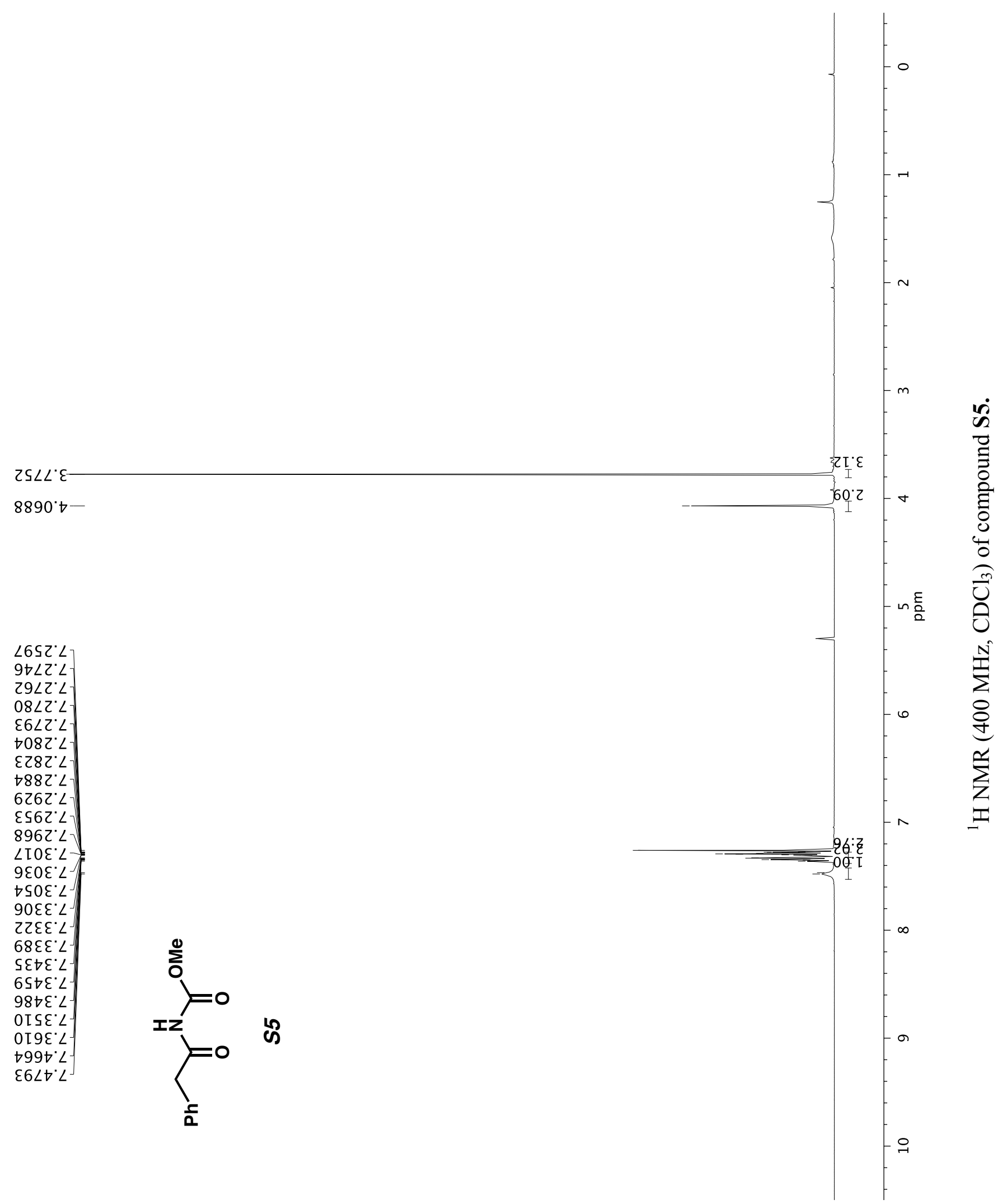


Wright, Haley, LaPointe, and Stoltz
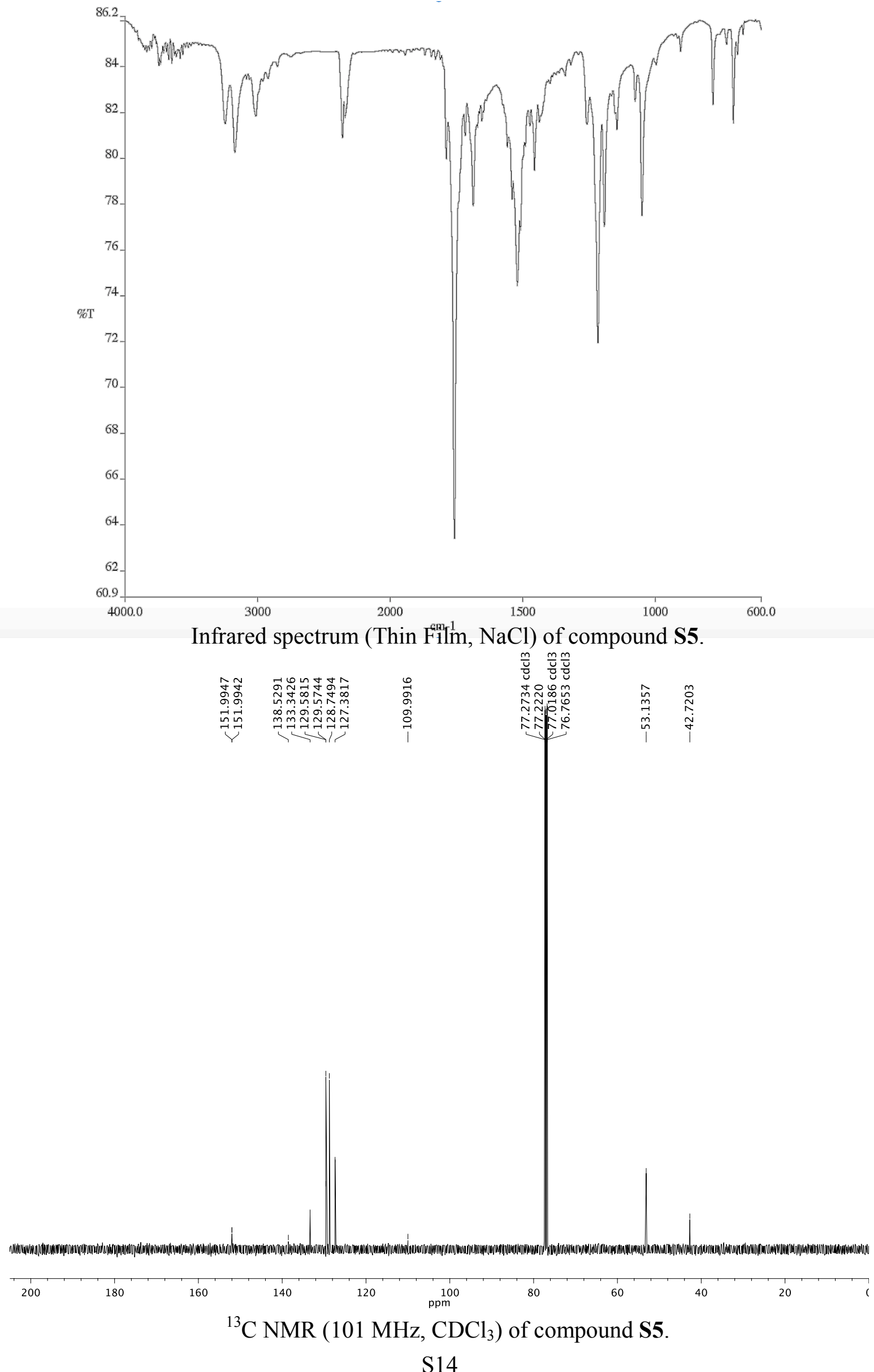


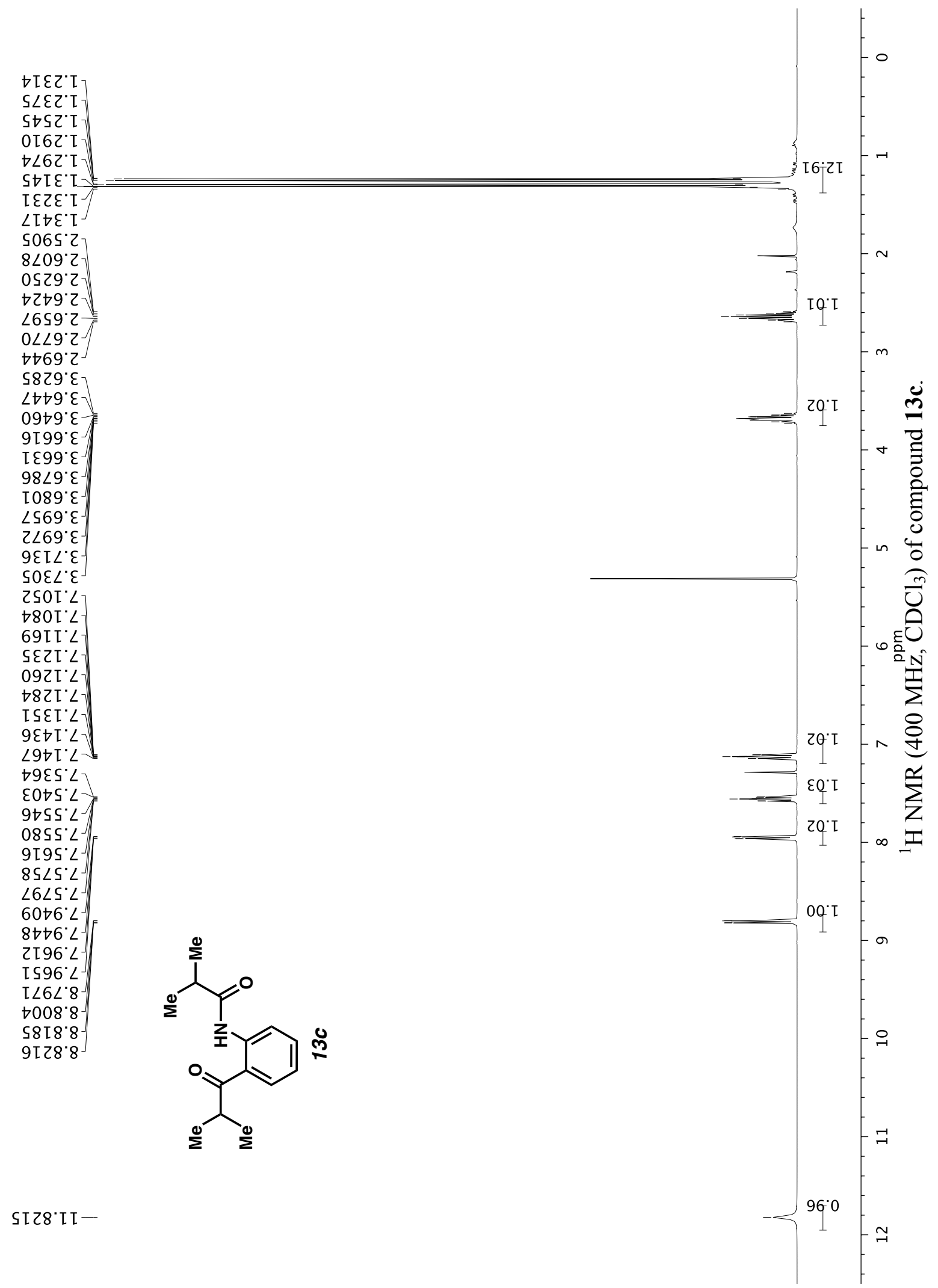




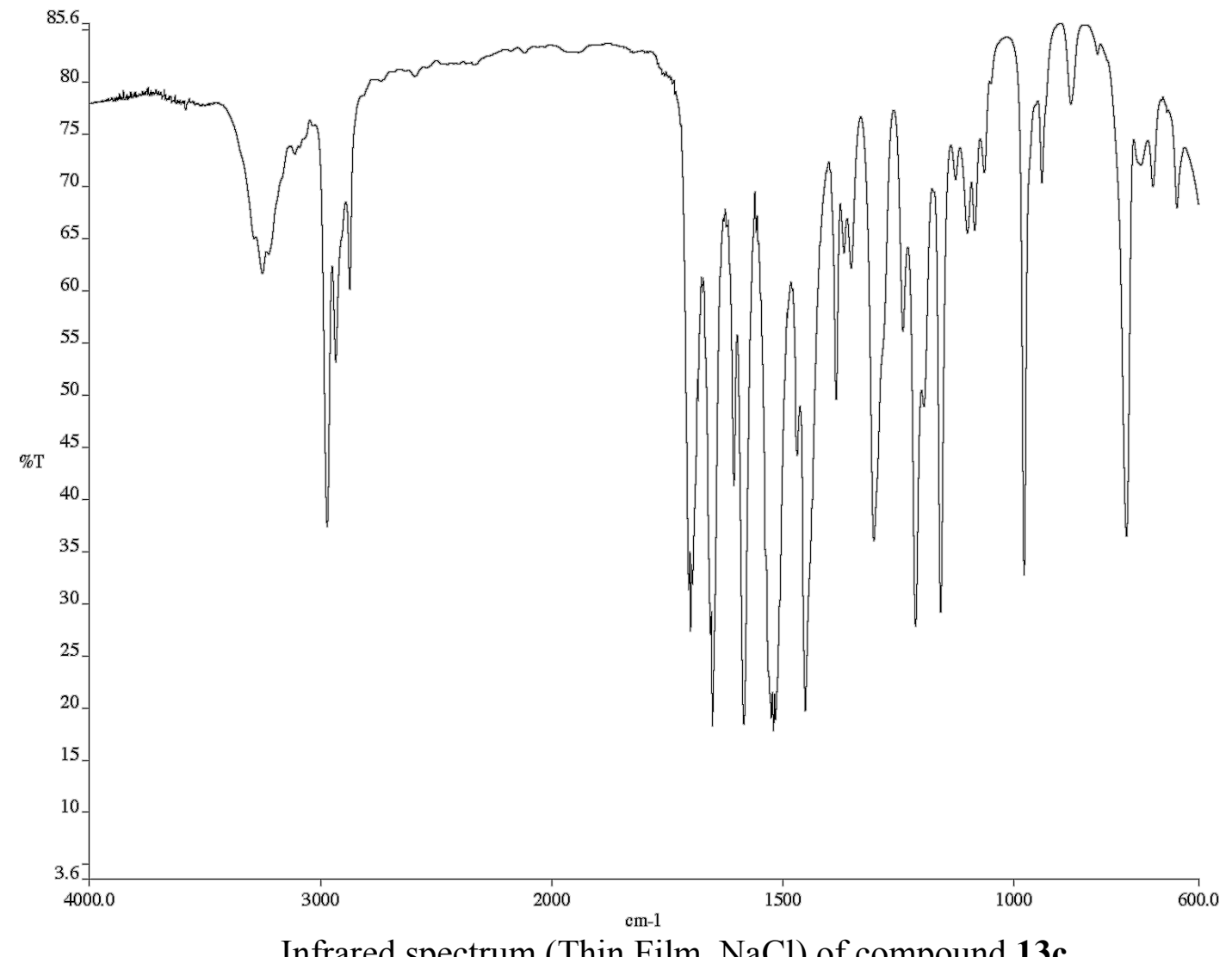

Infrared spectrum (Thin Film, $\mathrm{NaCl}$ ) of compound 13c.
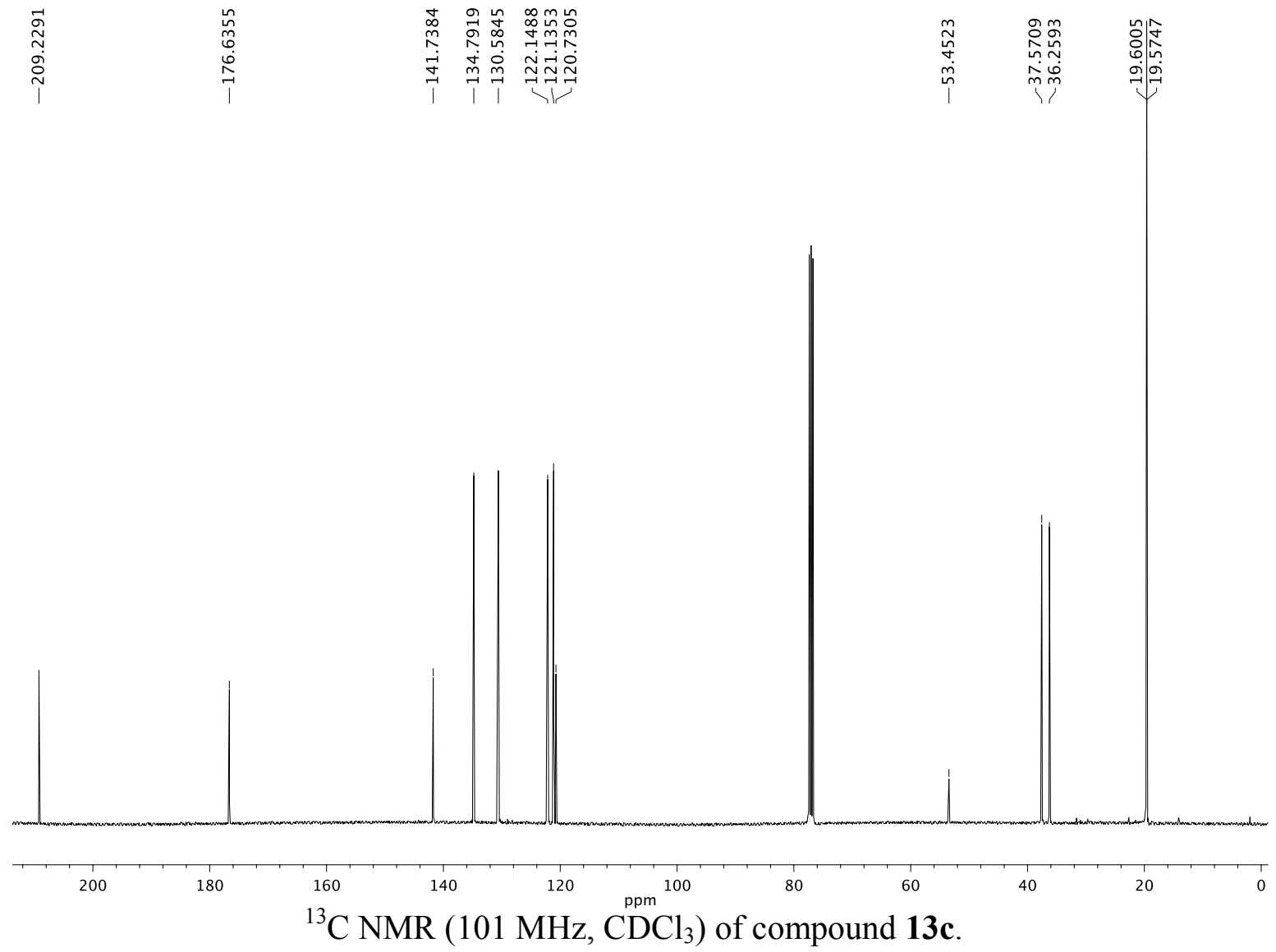


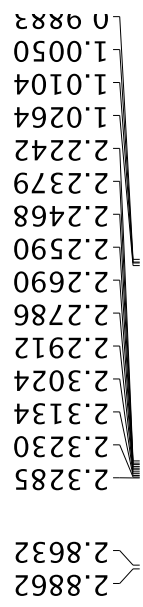

$0 \angle Z L \cdot I I-$

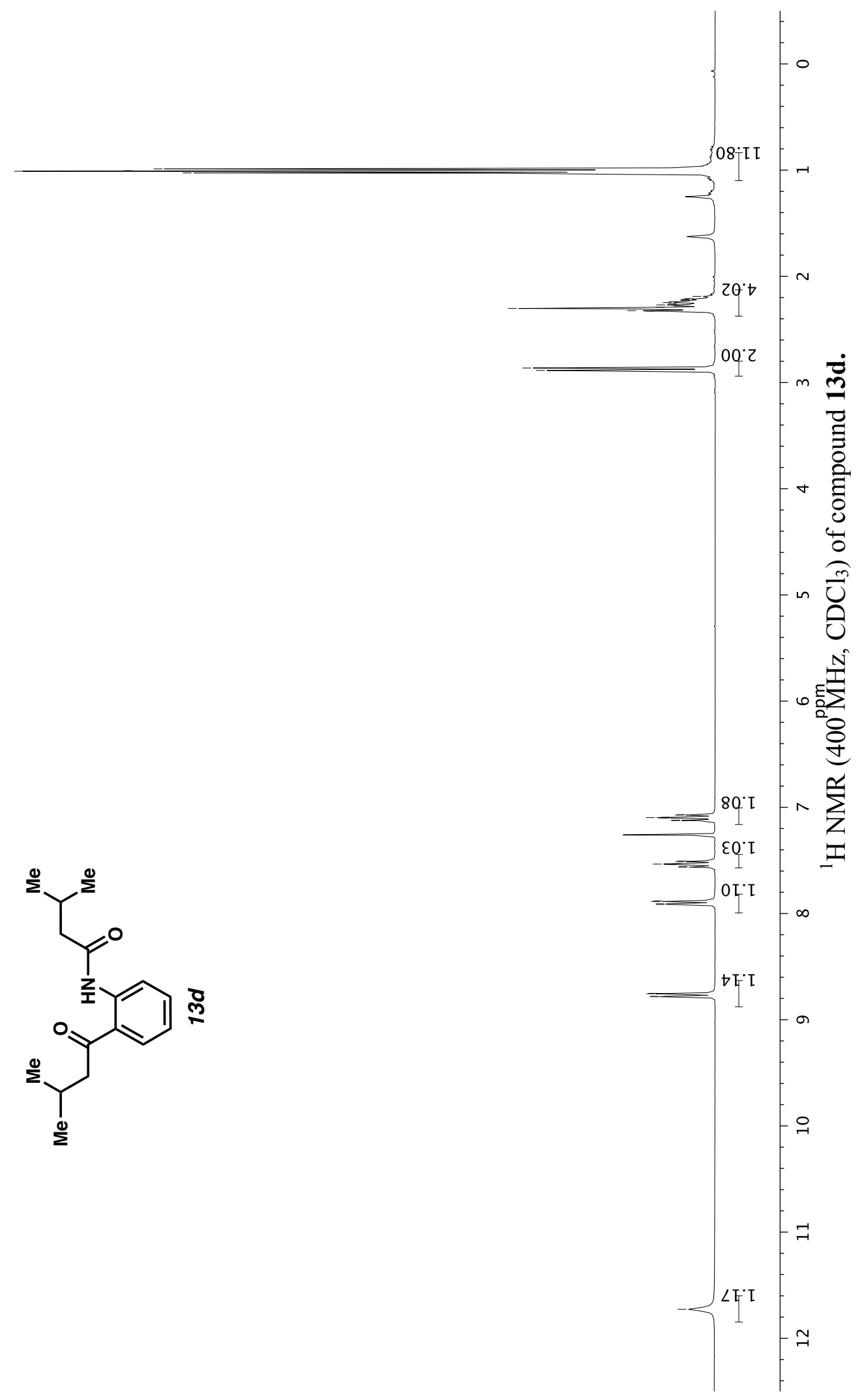



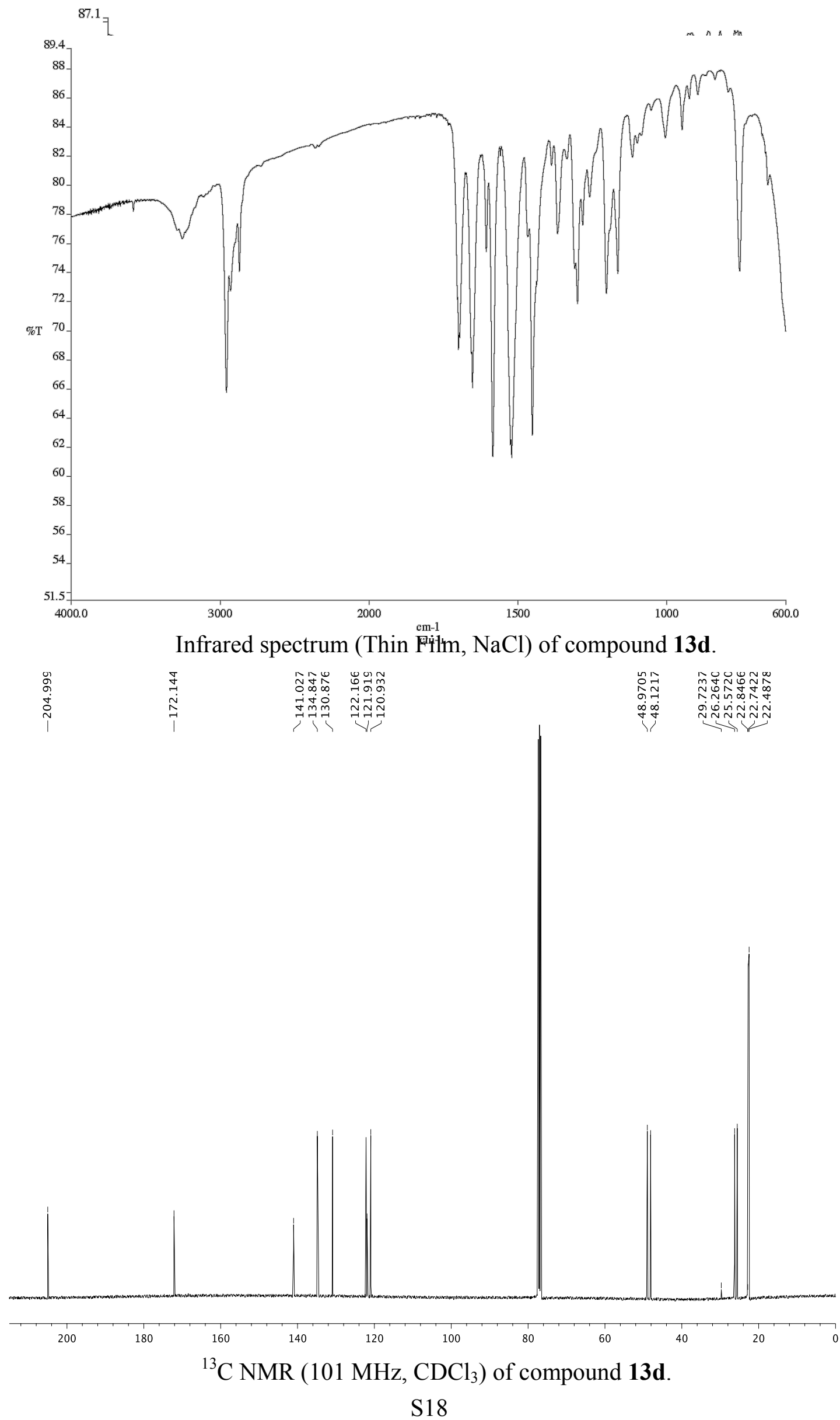

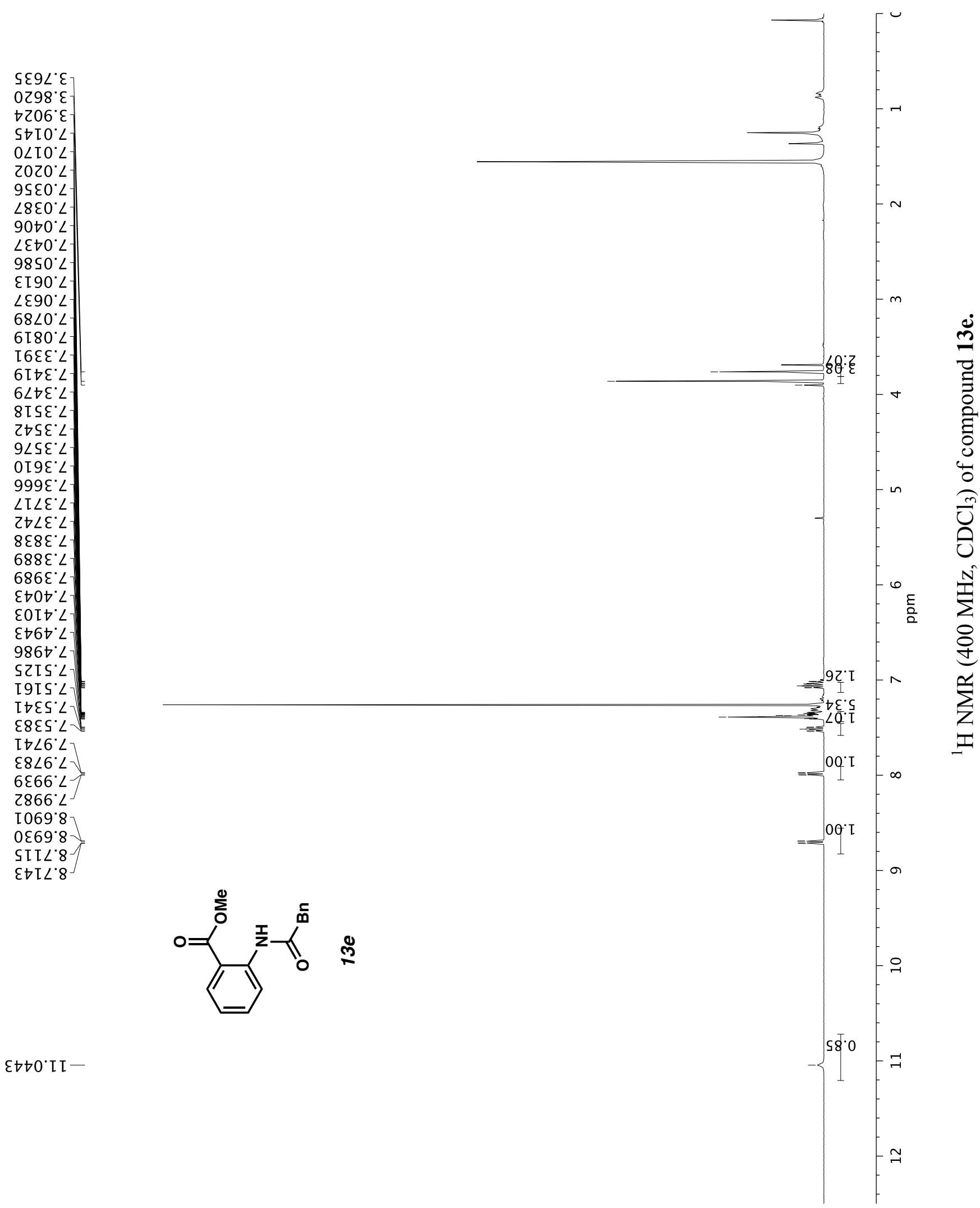

E†t0.II- 


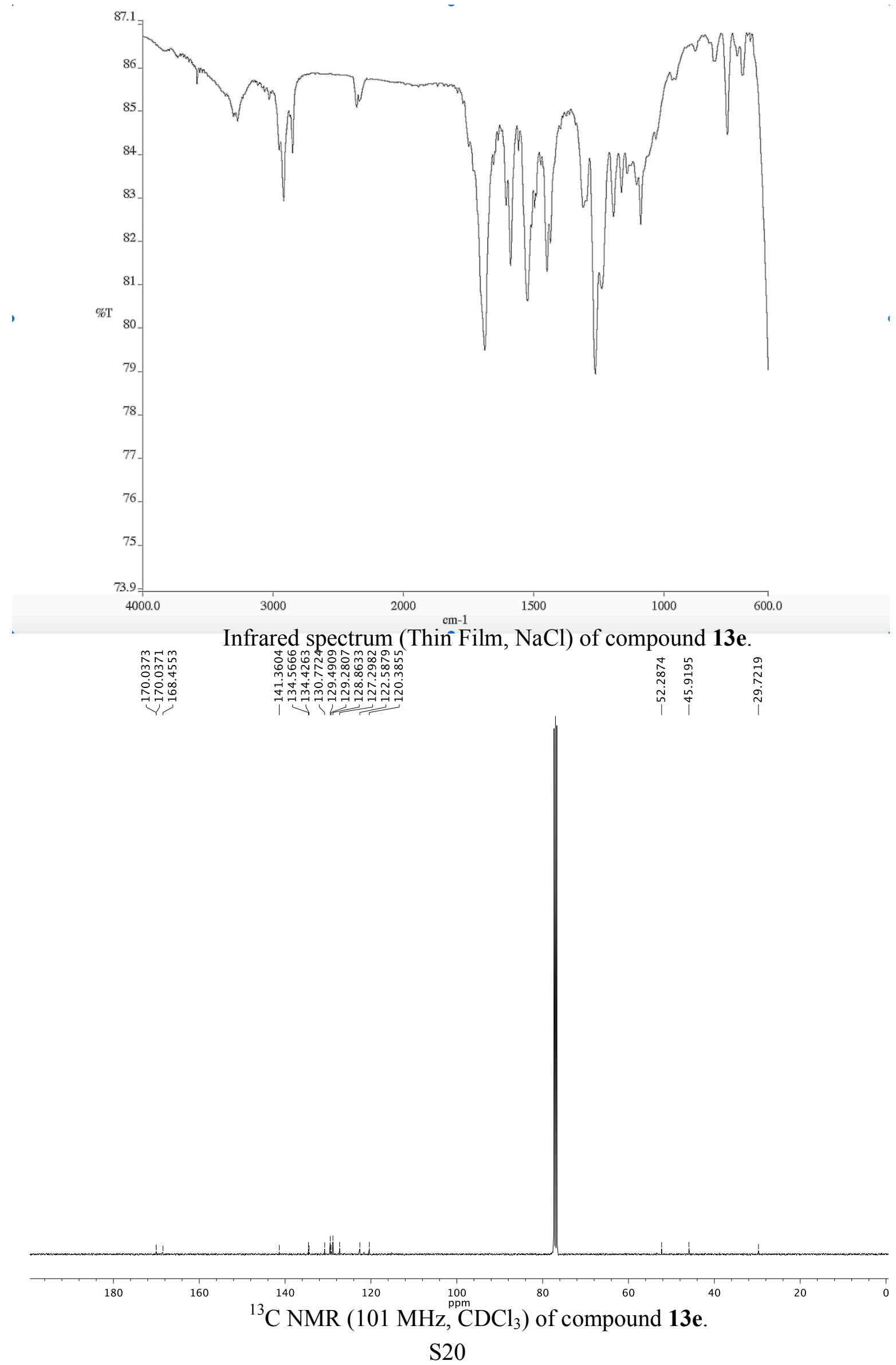




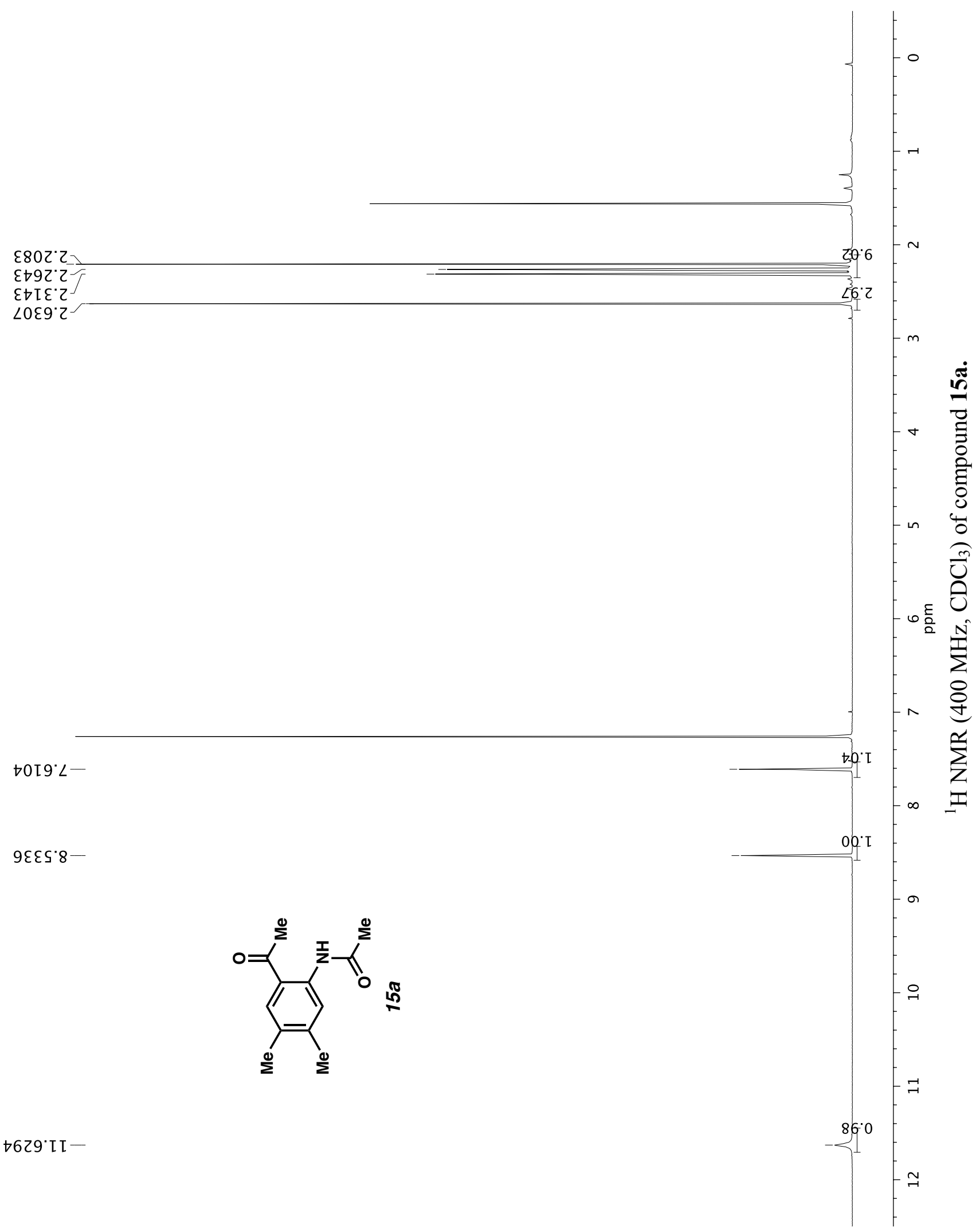



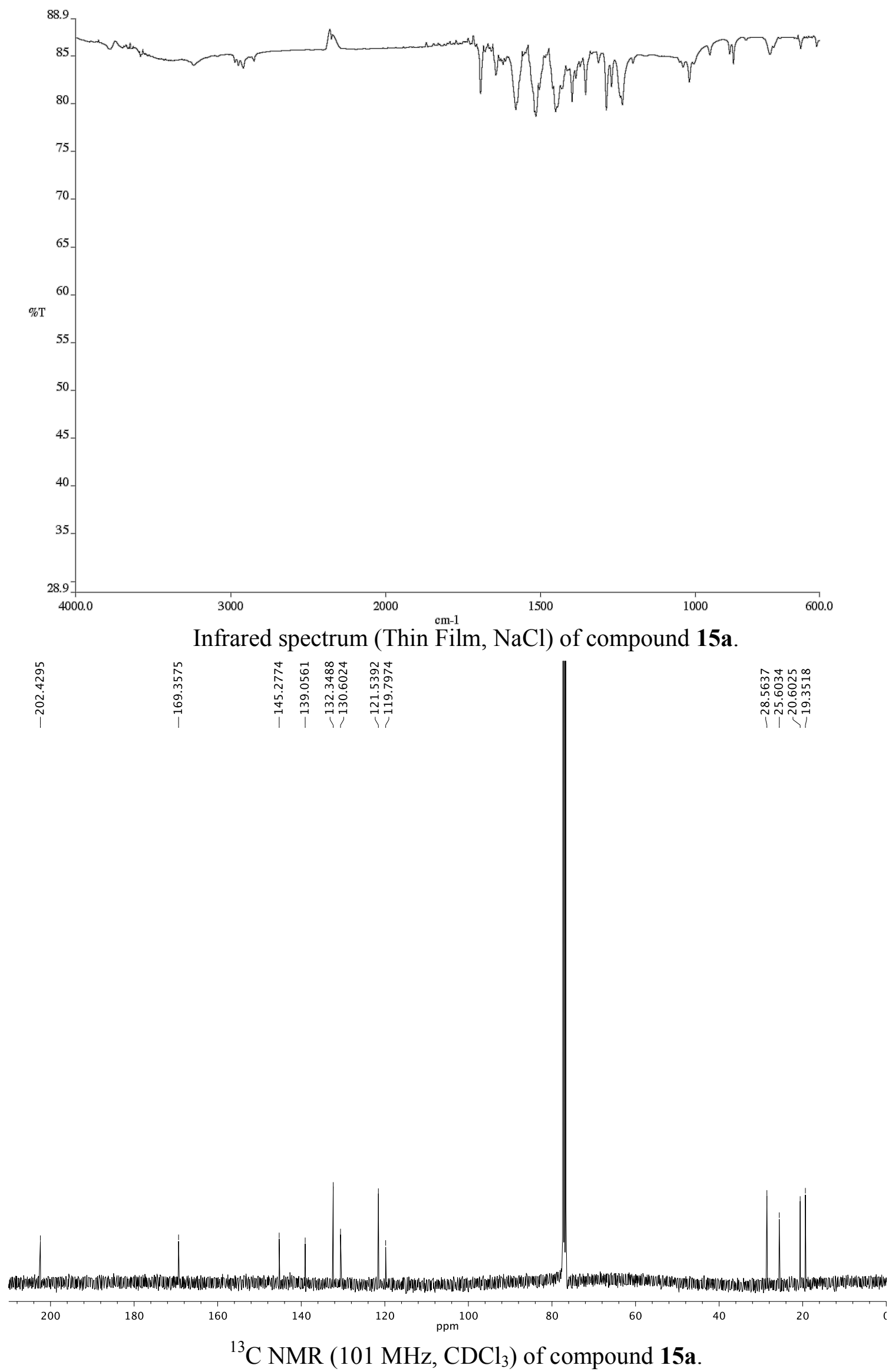


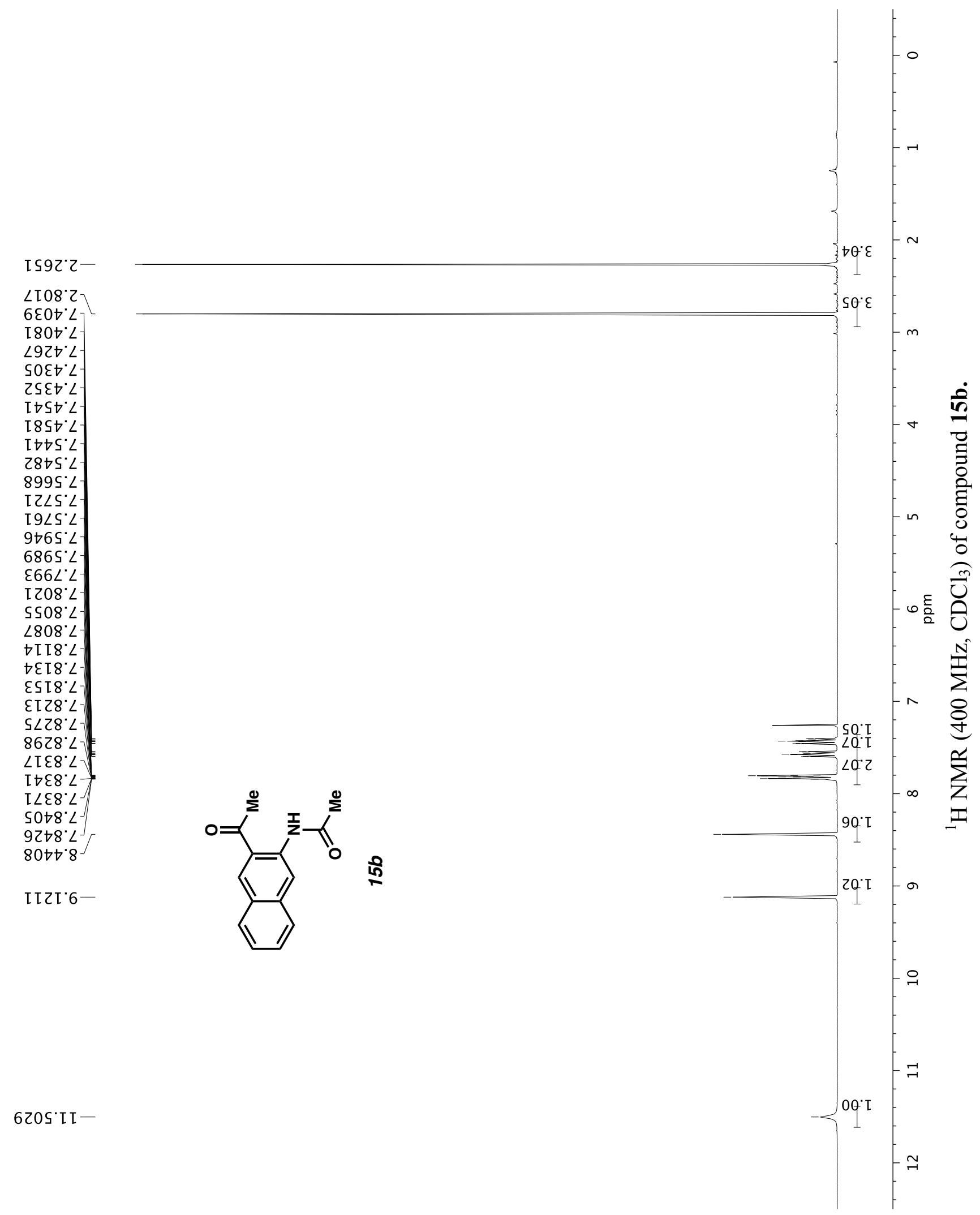



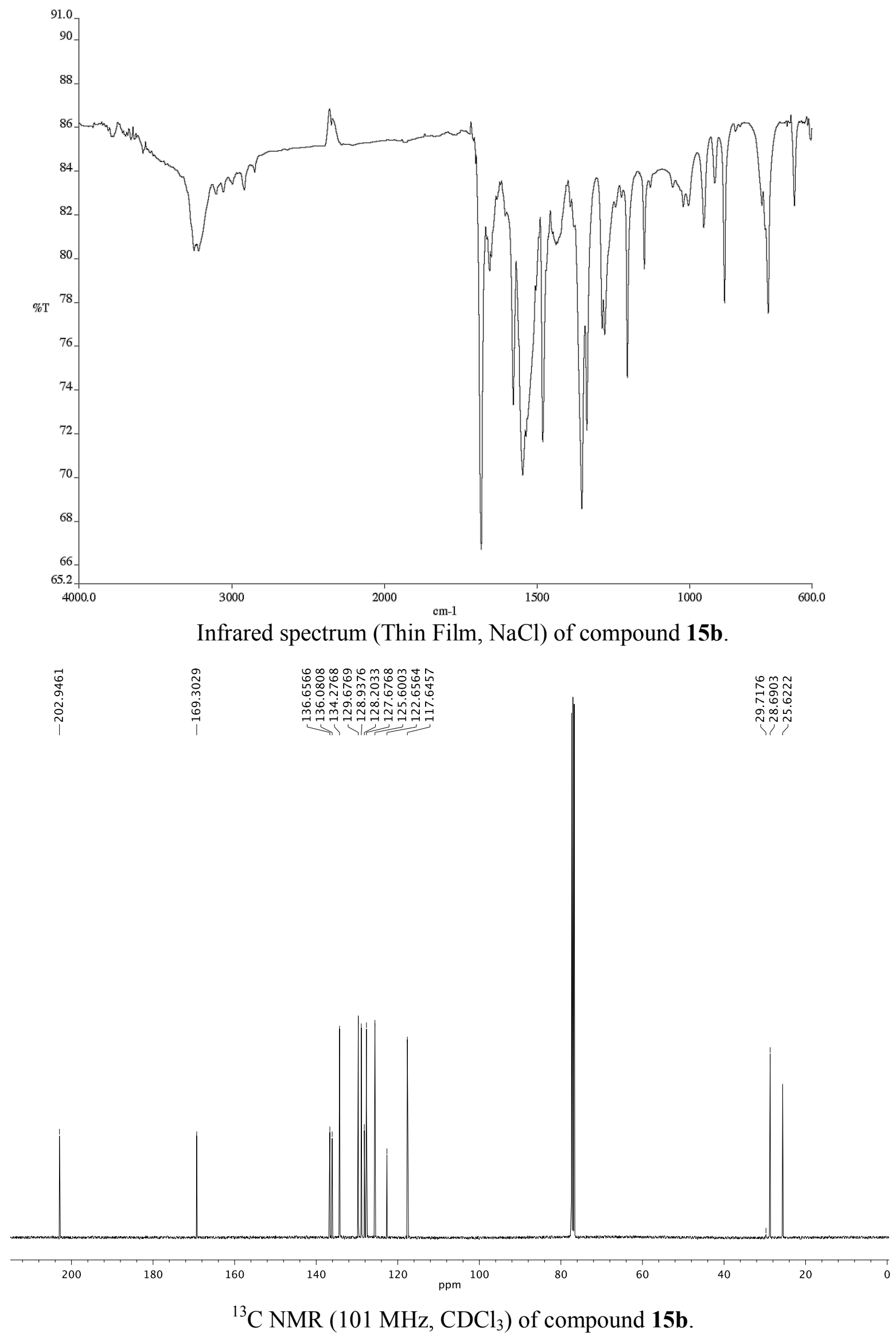


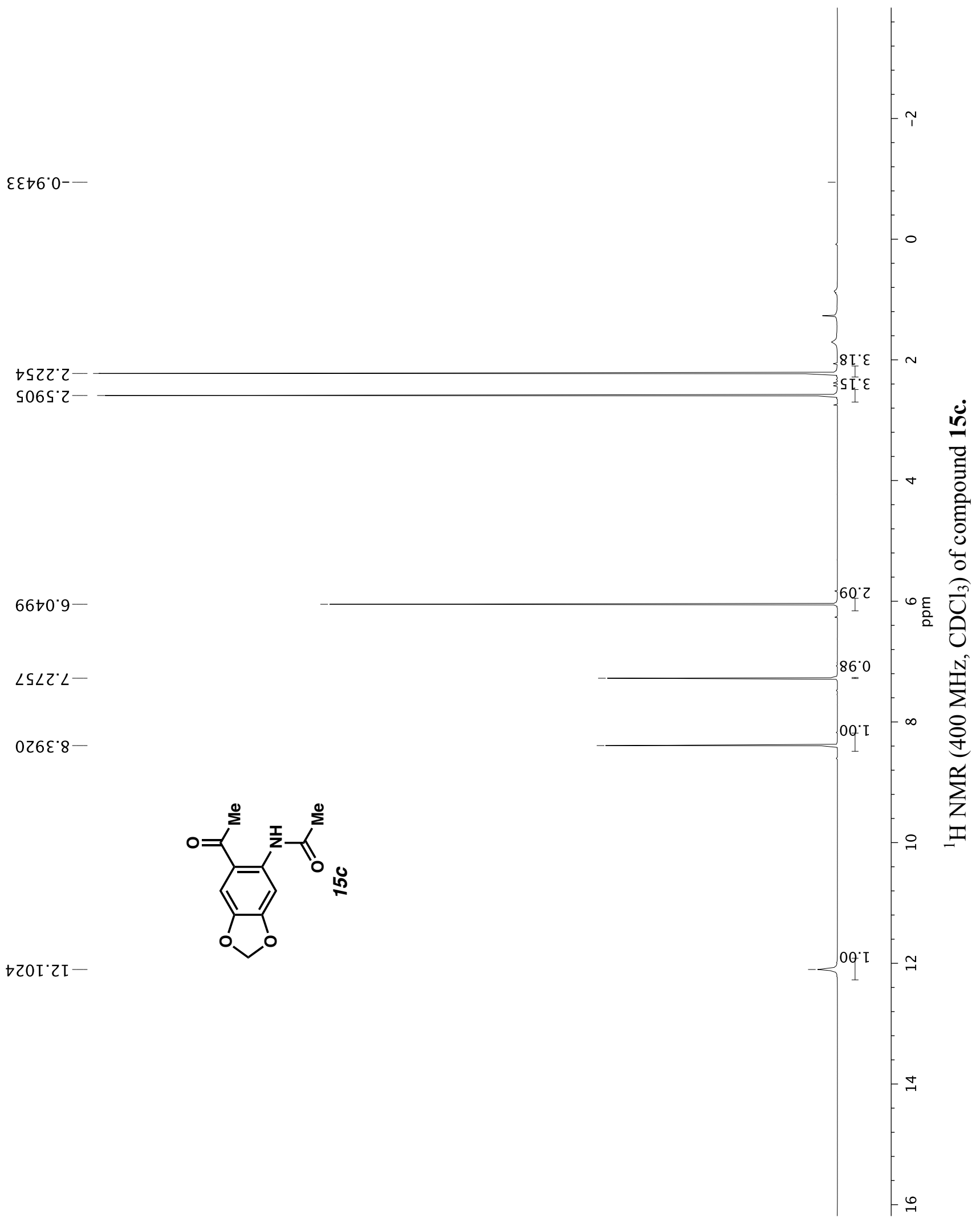




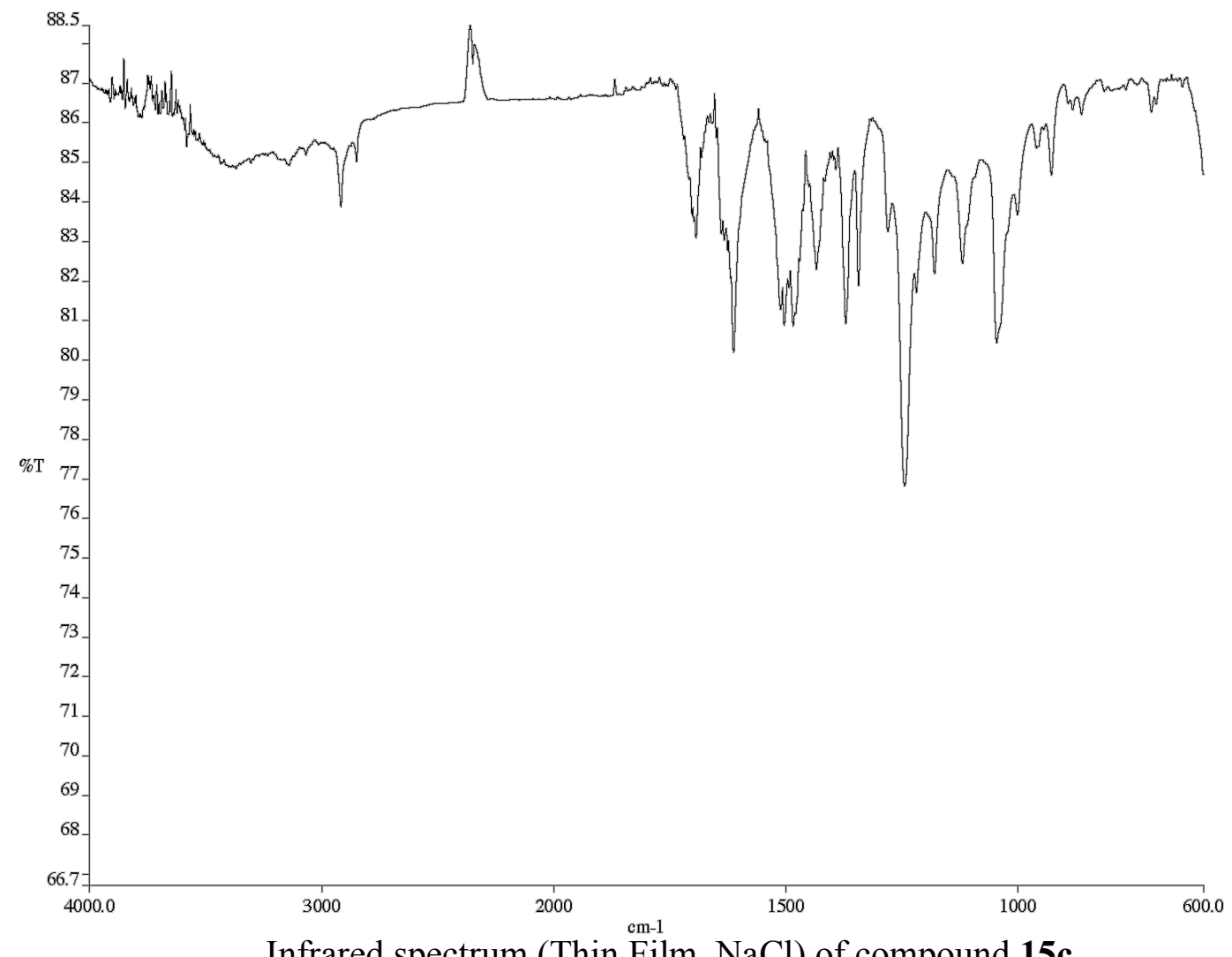

Infrared spectrum (Thin Film, $\mathrm{NaCl}$ ) of compound $\mathbf{1 5 c}$.

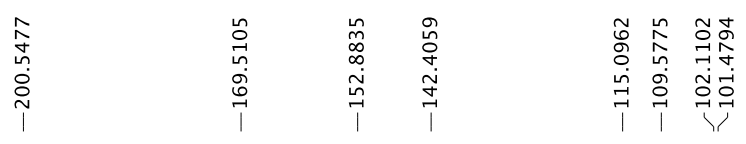

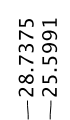

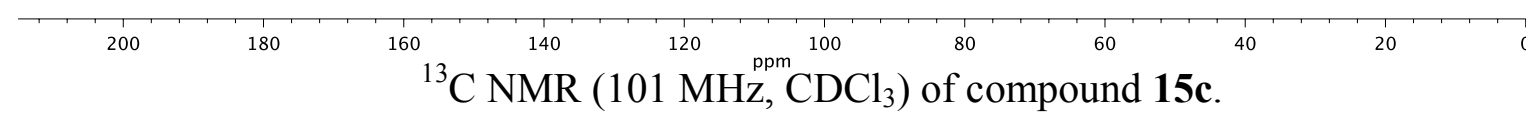
S26 


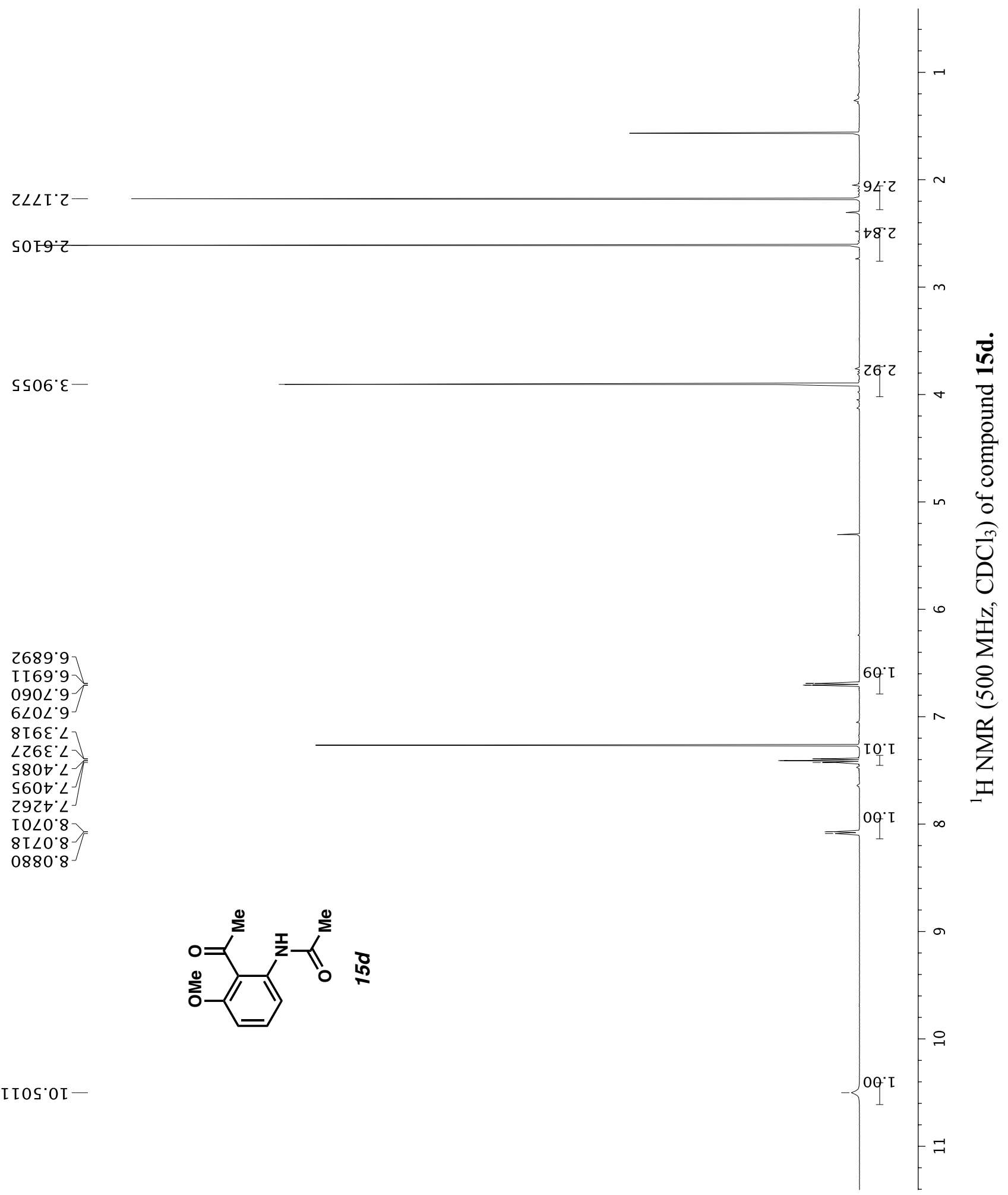



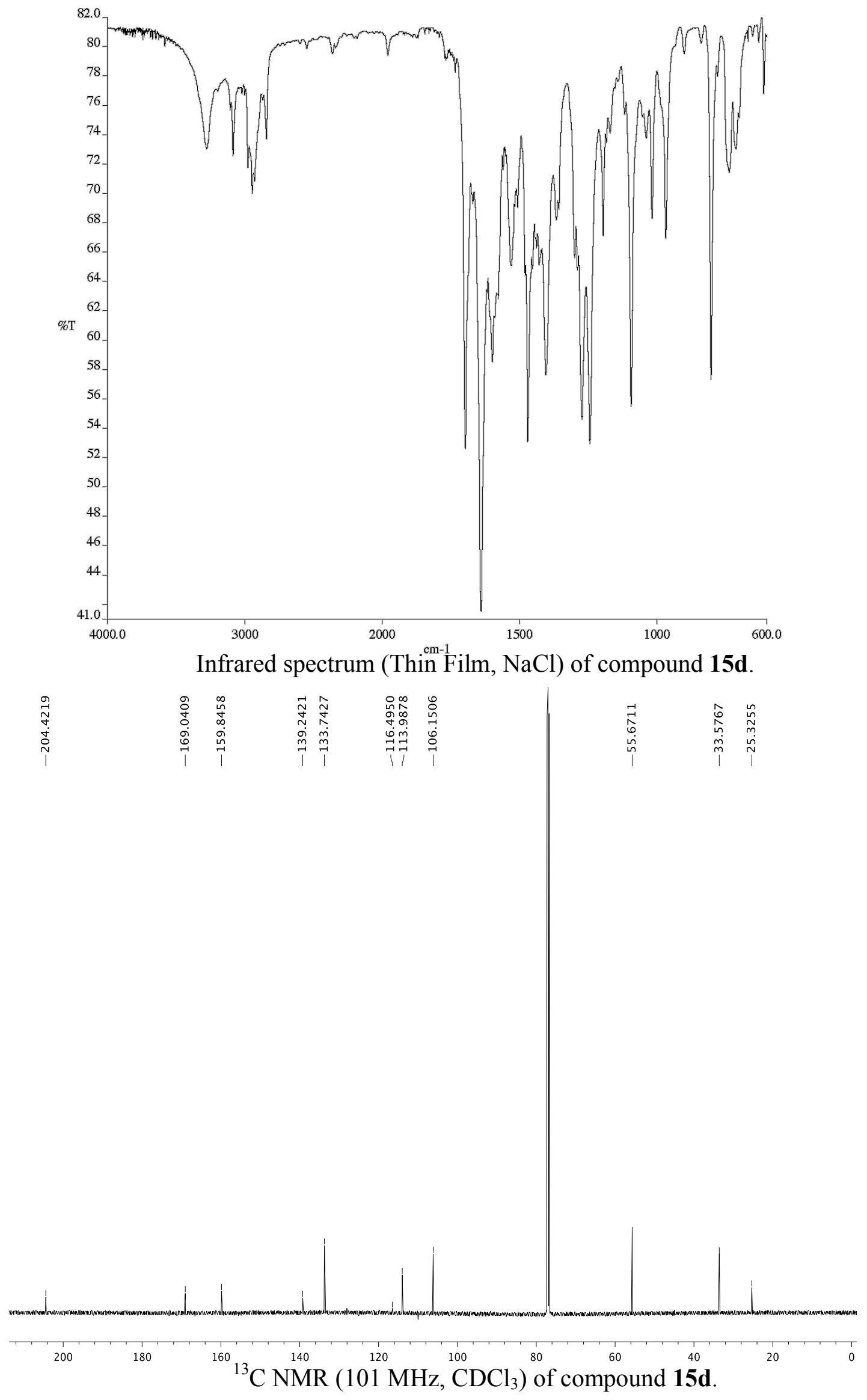

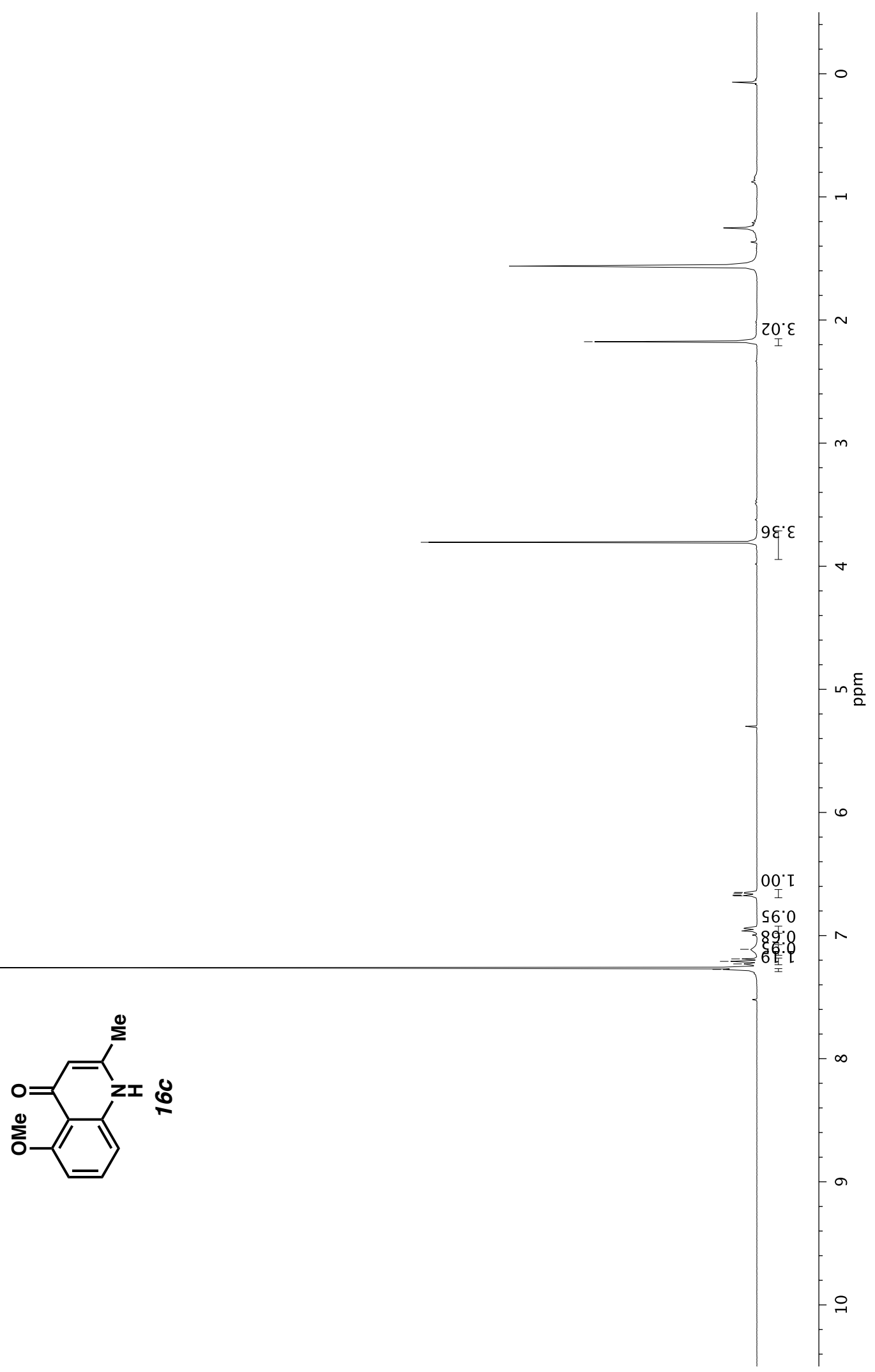


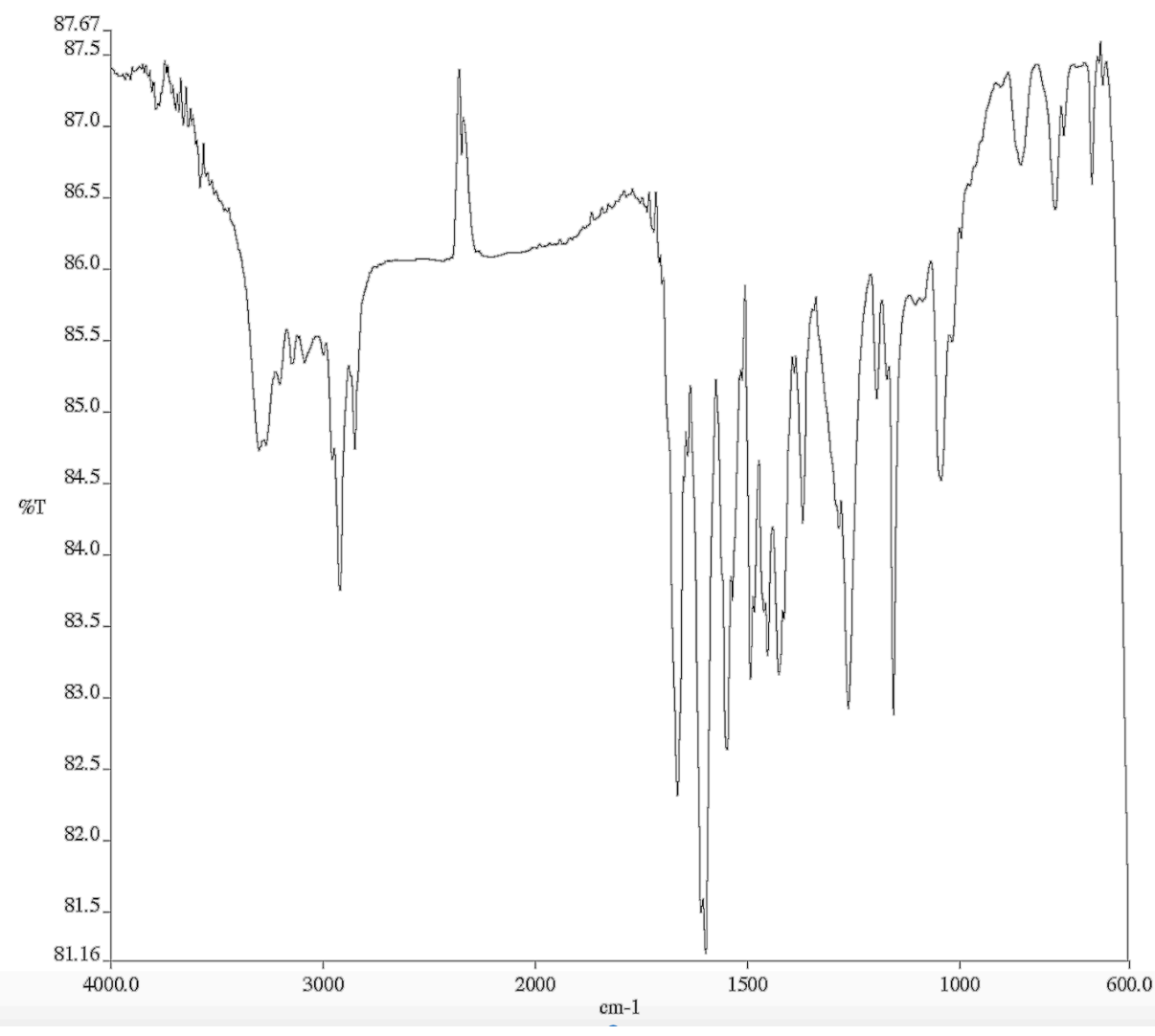

Infrared spectrum (Thin Film, $\mathrm{NaCl}$ ) of compound 16c.

\begin{tabular}{|c|c|c|c|}
\hline 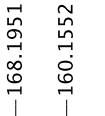 & 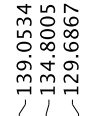 & 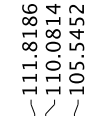 & 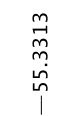 \\
\hline
\end{tabular}

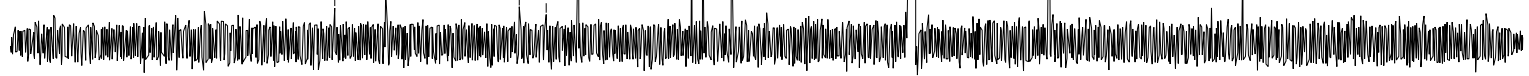

200

${ }^{13} \mathrm{C}^{160} \mathrm{NMR}\left(101 \mathrm{MHz}^{140}, \mathrm{CD}^{100} \mathrm{CP}_{3}\right)$ of compound 16c.

S30 
$\tau \angle 09^{\circ} Z$

$\angle O t \angle \cdot 9-$

ZSSE'L-

$S \nabla \angle \varepsilon^{\circ} \angle$

9t0t $\angle \square$

$\angle 025^{\circ} \mathrm{L}$

ZOSS: $\angle$

$\dagger 229^{\circ} \mathrm{L}$

$9929^{\circ} \mathrm{L}$

[OS $9^{\circ} \angle$

$08 \angle L \angle$

IS08. $L$
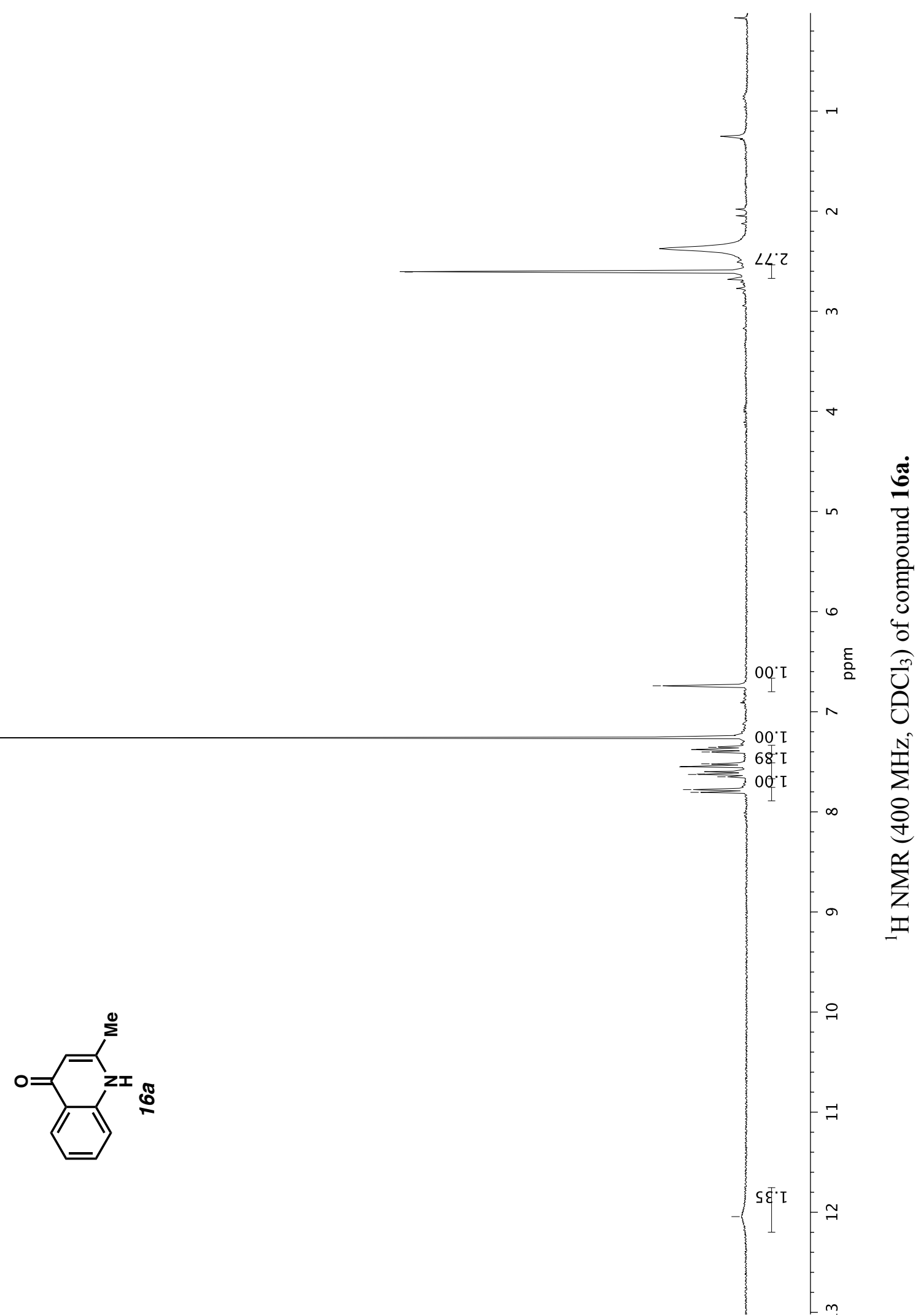


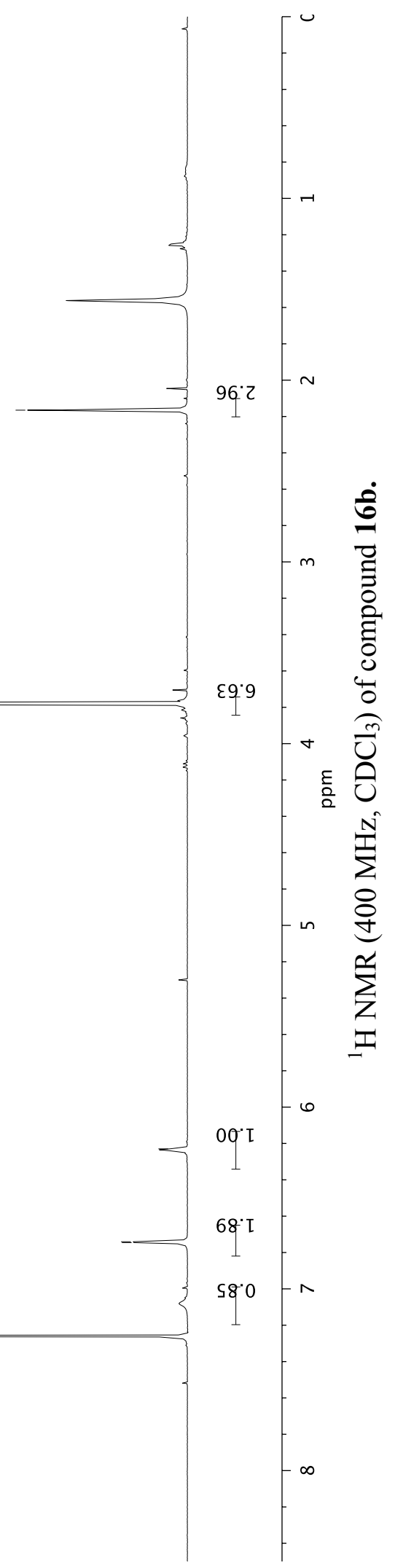

$\varepsilon 6 \angle L^{\circ} \varepsilon-$

$\angle 622^{\circ} 9-$

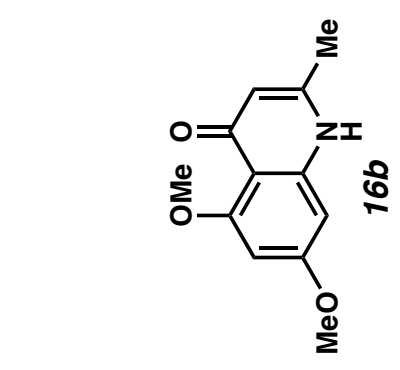

SOt $\angle$ ' 9

9StL' 9

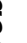

(2) 